\title{
Foundations for using linear temporal logic in Event-B refinement
}

\author{
Thai Son Hoang ${ }^{1}$, Steve Schneider ${ }^{2}$, Helen Treharne ${ }^{2}$ and David M. Williams ${ }^{3}$ \\ ${ }^{1}$ ECS, University of Southampton, Southampton, UK \\ ${ }^{2}$ Department of Computer Science, University of Surrey, Guildford, UK \\ ${ }^{3}$ VU University, Amsterdam, The Netherlands
}

\begin{abstract}
In this paper we present a new way of reconciling Event-B refinement with linear temporal logic (LTL) properties. In particular, the results presented in this paper allow properties to be established for abstract system models, and identify conditions to ensure that the properties (suitably translated) continue to hold as those models are developed through refinement. There are several novel elements to this achievement: (1) we identify conditions that allow LTL properties to be mapped across refinement chains; (2) we provide translations of LTL predicates to reflect the introduction through refinement of new events and the renaming and splitting of existing events; (3) we do this for an extended version of LTL particularly suited to Event-B, including state predicates and enabledness of events, which can be model-checked at the abstract level. Our results are more general than any previous work in this area, covering liveness in the context of anticipated events, and relaxing constraints between adjacent refinement levels. The approach is illustrated with a case study. This enables designers to develop event based models and to consider their execution patterns so that liveness and fairness properties can be verified for Event-B systems.
\end{abstract}

Keywords: Event-B, Refinement, Linear Temporal Logic

\section{Introduction}

Event-B [Abr10] is a step-wise development method with excellent tools: Atelier B [Cle14] and the Rodin platform $\left[\mathrm{ABH}^{+} 10, \mathrm{Z}^{+} 14\right]$ providing proof support and ProB [LB08] providing model checking. As Hoang and Abrial [HA11] clearly state the focus of verification within Event-B has been on the safety properties of a system to ensure that "something (bad) never happens". Typically, this has been done via the discharging of proof obligations. Nonetheless, the use of linear temporal logic (LTL) to specify temporal liveness properties has also been prevelant, for example in its application within the ProB tool [LFFP09]. The challenge is to identify more natural ways of integrating Event-B and LTL, so that LTL properties can be preserved by Event-B refinement, which is not the case in general.

Correspondence and offprint requests to: S. Schneider, E-mail: S.Schneider@surrey.ac.uk; H. Treharne, E-mail: H.Treharne@surrey.ac.uk 
Event-B describes systems in terms of machines with state, and events which are used to update the state. Events also have guards, which are conditions for the event to be enabled. One (abstract) machine may be refined by another (concrete) machine, using a refinement step. A linking invariant captures how the abstract and concrete states are related, and each abstract event must be refined by one or more concrete events whose state transformations match the abstract one in the sense of preserving the linking invariant. Refinement is transitive, so a sequence of refinement steps, known as a refinement chain, will result in a concrete machine which is a refinement of the original abstract one.

A particular feature provided by Event-B is the introduction of new events in a refinement step - events which do not refine any abstract event. This allows for refinements to add finer levels of granularity and concretisation as the design develops; there are many examples in [Abr 10]. These new events are invisible at the abstract level (they correspond to the abstract state not changing), and we generally need to verify that they cannot occur forever, to show that concrete progress corresponds (eventually) to progress at the abstract level. Event-B makes use of labels to keep track of the status of events as a refinement chain progresses. Event-B labels are anticipated, convergent and ordinary. The labelling of events in Event-B form part of the core of a system description but their inclusion is primarily to support the proof of safety properties and ensure that events cannot occur forever: convergent events must decrease a variant and anticipated events cannot increase it. In this paper, following Abrial [Abr10] all newly introduced events must be convergent or anticipated, and anticipated events must become convergent at some stage. As an initial example, consider a Lift machine with two events top and ground, representing movement to the top and to the ground floor. This can be refined by a machine Lift' introducing two new anticipated events openDoors and closeDoors. The events top and ground are blocked when the doors are open, but enabled when the doors are closed.

Linear temporal logic provides a specification language for capturing properties of executions of systems, and is appropriate for reasoning about liveness and fairness over entire system executions. For example, we might verify for Lift that whenever top occurs, then eventually ground will occur. However, this is not guaranteed for its refinement Lift': it may be that the doors open and close repeatedly forever following the top event, thus never reaching the next ground event. Alternatively it may be that the system deadlocks with the doors open, again preventing ground from occurring. Both of these possibilities are permitted within the Event-B refinement framework. Hence we see that LTL properties are not automatically preserved by Event-B refinement. In the first case we would require some assurance that openDoors and closeDoors cannot repeat forever without the lift moving; in the second case we require some fairness property on closeDoors to prevent termination with the doors open.

In this paper we present results to enable temporal logic properties to be carried through Event-B refinement chains. The results generalise to events that are split-refined by several events - during a refinement chain. The paper builds on [STWW14b] but it is entirely self-contained and provides significant theoretical extension to previous work. Importantly, the results presented in this paper support discharging a wider range of LTL properties through Event-B refinement than the previous work, since we can now include all the LTL operators supported by the ProB tool, i.e., support for the next, proposition and enabled operators is new for this paper. Note that LTL properties can be introduced at different levels of refinement. This paper's focus is much more in clarifying what refinement proofs need to be conducted during a refinement depending on the LTL properties of interest. The main new contribution is the result that allows properties that include the enabled $(e(t))$ operator to be preserved through a refinement chain and this requires a new proof obligation to be discharged during development.

The benefit of the new extensions is that they have facilitated the removal of an awkward restriction $(\beta$ dependency), which identified conditions on temporal logic properties to make them suitable for use in a refinement chain. Hence the LTL properties that can be specified are much more natural than in [STWW14b]. The rigour of the results is underpinned by our process algebra understanding of the Event-B semantics, in particular the traces, divergences and infinite traces semantics used for CSP and applied to Event-B in [STW14].

The paper is organised as follows: Sect. 2 provides the necessary Event-B refinement background and one of the refinement strategies we use in the paper and was previously presented in [STWW14b]. Section 3 introduces a running example and is modified from the example in [STWW14b] to include more detail and provide the basis for new LTL properties. Section 4 introduces the infinite traces Event-B semantics required as a basis for the technical results. It is an extension of our previous work since a new definition of the refinement relation is required to support the proofs. Section 5 defines the LTL we use and it is an extended version of LTL from that presented in [STWW14b]. In particular the extended LTL contains the next operator, state predicates and supports the enabled operator. Sections 6 and 7 present and illustrate the main theoretical results. The proofs of these results are included in the Appendix. The sections have been split into two so that it is clear what refinements 
proof obligations would need to be carried out in practice for a particular system in order to discharge appropriate LTL properties. The proof obligations required to be discharged for a particular system are dependent on the operators used within the LTL properties. Section 6 is significantly different from our referenced conference paper and Sect. 7 is entirely new. We place our work into the context of related work in Sect. 8 and in particular [HH13]. The paper concludes with a discussion and directions for future work in Sect. 9.

\section{Event-B}

\subsection{Event-B machines}

An Event-B development is defined using machines. A machine $M$ contains a set of variables $v$ and a set of events. The alphabet of $M, \alpha M$, is the set of events defined in $M$. Each event evt has the general form evt $\hat{=}$ any $x$ where $G_{e v t}(x, v)$ then $v: \mid B A_{e v t}\left(v, x, v^{\prime}\right)$ end, where $x$ represents the parameters of the event, the guard $G_{e v t}(x, v)$ is the condition for the event to be enabled. The body is given by $v: \mid B A_{\text {evt }}\left(v, x, v^{\prime}\right)$ whose execution assigns to $v$ any value $v^{\prime}$ which makes the before-after predicate $B A_{\text {evt }}\left(v, x, v^{\prime}\right)$ true. This simplifies to evt $\hat{=}$ when $G_{e v t}(v)$ then $v: \mid B A_{\text {evt }}\left(v, v^{\prime}\right)$ end when there are no parameters, since the guard and the before-after predicate does not refer to the parameters $x$. In the paper we also use the shorthand $G_{e v t}$ to denote the guard of the event evt when we omit the parameters for brevity. In practice, events are written using the Generalised Substitution Language, a syntactic sugar for events which encapsulate before-after relationships in a programming style by means of abstract assignments of values to variables. For example, $v:=v+x$ corresponds to the relation $v^{\prime}=v+x$.

Variables of a machine are initialised in an initialisation event init and are constrained by an invariant $I(v)$. The Event-B approach to semantics is to associate proof obligations with machines. The key proof obligation, INV, is that all events must preserve the invariant. There is also a proof obligation on a machine with respect to deadlock freedom which means that at least one event in $M$ is enabled at anytime. When this obligation holds $M$ is deadlock free.

\subsection{Event-B refinement}

An Event-B development is a sequence of machines linked by a refinement relationship. In this paper we use $M$ and $M^{\prime}$ when referring to a refinement between an abstract machine $M$ and a concrete machine $M^{\prime}$ whereas a chain of refinements is referred to using numbered subscripts, i.e., $M_{0}, M_{i}, \ldots, M_{n}$, to represent the specific refinement levels.

A machine $M$ is considered to be refined by $M^{\prime}$ if the given linking invariant $J$ on the variables between the two machines, $J\left(v, v^{\prime}\right)$, is established by their initialisation, and preserved by all events. This requirement is captured by the INV_REF proof obligation. Formally, we denote the refinement relation between two machines, written $M \preccurlyeq M^{\prime}$, when all the following proof obligations hold for every event: feasibility FIS_REF, guard strengthening GRD_REF and invariant preservation INV_REF. Feasibility of an event is the property that, if the event is enabled (i.e., the guard is true), then there is some after-state. Guard strengthening requires that when a concrete event is enabled, then so is the abstract one. Finally, simulation ensures the occurrence of events in the concrete machine can be matched in the abstract one (including the initialization event). Further details of these proof obligations can be found in [Abr10].

Refinement is transitive, therefore for a chain of refinements $M_{0} \preccurlyeq M_{1} \preccurlyeq \cdots \preccurlyeq M_{n}$ there is a linking invariant between each refinement step such that there is a combination of linking invariants for all refinement steps: We make explicit that there is a relationship between the states of the initial and final machine through a chain of refinements: $\mathcal{J}\left(v_{0}, v_{n}\right)=\exists v_{1}, v_{2}, \ldots v_{n-1} . J_{1}\left(v_{0}, v_{1}\right) \wedge J_{2}\left(v_{1}, v_{2}\right) \cdots \wedge J_{n}\left(v_{n-1}, v_{n}\right)$, and similarly between two machines $M_{i}$ and $M_{j}$ where $j>i$ in a refinement chain as follows: $\mathcal{J}\left(v_{i}, v_{j}\right)=\exists v_{i+1}, \ldots v_{j-1} . J_{i+1}\left(v_{i}, v_{i+1}\right) \cdots \wedge$ $J_{j}\left(v_{j-1}, v_{j}\right)$, where $M_{i}$ and $M_{j}$ are adjacent $\mathcal{J}\left(v_{i}, v_{j}\right)=J\left(v_{i}, v_{j}\right)$.

A refinement machine can introduce new events and split existing events. We omit the treatment of merging events in this paper. New events $e v t^{\prime}$ are treated as refinements of skip, i.e., evt' does not refine an event in $M$. Note that when splitting events, $M^{\prime}$ has several events $e v t_{1}^{\prime} \ldots e v t_{j}^{\prime}$ refining a single event evt. 


\begin{tabular}{|c|c|c|c|c|}
\hline \multirow{7}{*}{$\begin{array}{l}\text { selectItem (o) } \\
\text { dispenseItem (o) }\end{array}$} & selectBiscuit (o) & selectBiscuit (o) & selectBiscuit (o) & selectBiscuit (o) \\
\hline & selectChoc (o) & selectChoc (o) & selectChoc (o) & selectChoc (o) \\
\hline & dispenseBiscuit (o) & dispenseBiscuit (o) & dispenseBiscuit (o) & dispenseBiscuit (o) \\
\hline & dispenseChoc (o) & dispenseChoc (o) & dispenseChoc (o) & dispenseChoc (o) \\
\hline & & pay (a) & pay (a) & pay (c) \\
\hline & & refund (c) & refund (o) & refund (o) \\
\hline & & & refill (c) & refill (o) \\
\hline$V M_{0}$ & $V M_{1}$ & $V M_{2}$ & $V M_{3}$ & $V M_{4}$ \\
\hline
\end{tabular}

Fig. 1. Events and their annotations in the vending machine development

$$
\begin{aligned}
f 1 & =\{s b \mapsto s i, s c \mapsto s i, d b \mapsto d i, d c \mapsto d i\} \\
f 2 & =\{s b \mapsto s b, s c \mapsto s c, d b \mapsto d b, d c \mapsto d c\}=i d \\
f 3 & =\{s b \mapsto s b, s c \mapsto s c, d b \mapsto d b, d c \mapsto d c, p a y \mapsto \text { pay, refund } \mapsto \text { refund }\} \\
f 4 & =\{s b \mapsto s b, s c \mapsto s c, d b \mapsto d b, d c \mapsto d c, p a y \mapsto \text { pay, refund } \mapsto \text { refund, refill } \mapsto \text { refill }\} \\
g_{1,4} & =f_{4} ; f_{3} ; f_{2} ; f_{1}=\{s b \mapsto s i, s c \mapsto s i, d b \mapsto d i, d c \mapsto d i\}
\end{aligned}
$$

Fig. 2. Function mappings for the vending machine development

We define a function $f$ which maps each concrete event to the abstract event that it refines. Therefore, the type of $f$ is $f \in \alpha M^{\prime} \rightarrow \alpha M$, a partial surjection from the alphabet of the refinement machine $M^{\prime}$ onto the alphabet of the abstract machine $M$. New concrete events will not map to any abstract event and are therefore not in $\operatorname{dom}(f)$; this is why $f$ is a partial function. In the general case $f$ will be many-to-one, since many concrete events may map to a single abstract event. Thus, $f\left(e v t_{1}^{\prime}\right)=e v t \Leftrightarrow e v t_{1}^{\prime}$ refines evt and similarly for any other refinement events which relate to evt. In this paper we include the refines annotation only when we are splitting events in a refinement, otherwise we consider all similarly named events in a refinement step to be straightforward refinements of their counterparts in the previous step.

We also provide a clear definition, Definition 2.1, to relate the mapping of concrete events to abstract events through a refinement chain. The function $g_{i, j}$ defines a mapping for concrete events to abstract events, made up of composing the function mappings $f$ at each refinement level, i.e., $f_{j}$ down to $f_{i}$, where $i \leq j$. We explicitly annotate $f$ with the appropriate refinement level, i.e., $f_{i+1}: \alpha M_{i+1} \rightarrow \alpha M_{i}$ (Note that $g_{1,1}=f_{1}$ ). $g_{i, j}$ is also a partial surjective function. Note that the annotated index is the one from the concrete machine that $f$ is mapping from. Thus for an arbitrary chain we define the compositional mapping as follows:

Definition $2.1 g_{i, j}=f_{j} ; f_{j-1} ; \ldots ; f_{i}$

Note that $f_{j} ; f_{j-1} ; \ldots ; f_{i}=f_{i} \circ \ldots \circ f_{j-1} \circ f_{j}$. Also $g_{1, n}$ represents the compositional mapping for the entire refinement chain $M_{0}$ to $M_{n}$. Observe that $g_{i, n}$ will be undefined on any event that does not map to the first machine $M_{i-1}$ in the sequence. In other words any event that was introduced at some point in the refinement chain does not map back to $M_{i-1}$.

Figure 1 illustrates a vending machine development, detailed in Sect. 3 that we use throughout the paper and Fig. 2 identifies the functional mappings between each refinement layer within that development (using abbreviations for the select and dispense events, for example selectBiscuit is $s b$ ).

In Sect. 1 we introduced the three kinds of labelling of events in Event-B: anticipated (a), convergent (c) and ordinary (o) and noted that convergent events are those which must not execute forever whereas anticipated events provide a means of deferring consideration of divergence-freedom until later refinement steps. The proof obligation that deals with divergences is WFD_REF. It requires that the proposed variant $V$ of a refinement machine satisfies the appropriate properties: that it is a natural number, that it decreases on occurrence of any convergent event, and that it does not increase on occurrence of any anticipated event. Alternatively a variant can be a finite set that decreases in size with convergent events, and does not increase with anticipated events. Therefore, we augment the previous refinement relation with WFD_REF such that $M \preccurlyeq W M^{\prime}$. Ordinary events can occur forever and therefore WFD_REF is not applicable for such events. Note that in Sect. 7 we will introduce a further stronger notion of refinement that takes liveness into account. 


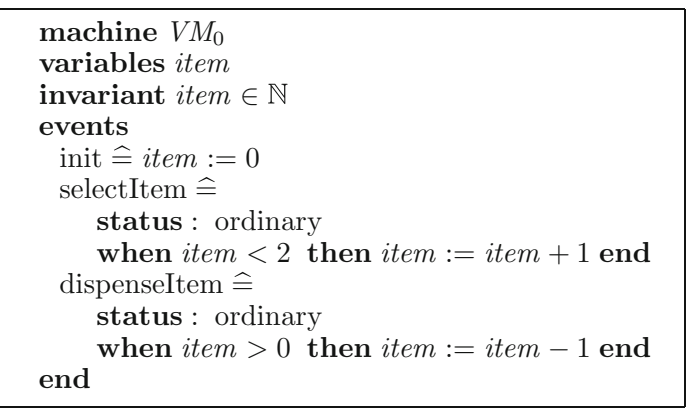

Fig. 3. $V M_{0}$

\subsection{Event-B development strategy}

Event-B has a strong but flexible refinement strategy which is described in [HLP13]. In [STW14] we also discussed different Event-B refinement strategies and characterised them with respect to the approaches documented by Abrial in [Abr10] and supported by the Rodin tool. In this paper we focus on the simplest strategy, and the one most commonly used. It is also a strategy supported by the Atelier B tool [Cle14]. The strategy has the following set of restrictions on a refinement chain $M_{0} \preccurlyeq{ }_{W} M_{1} \preccurlyeq{ }_{W} \cdots \preccurlyeq{ }_{W} M_{n}$ :

1. all events in $M_{0}$ are labelled ordinary. This set of events is referred to as $O_{0}$.

2. each event of $M_{i}$ is refined by at least one event of $M_{i+1}$ (i.e., events can be split);

3. each new event in $M_{i}$ is either anticipated or convergent, where $i>0$;

4. each event in $M_{i+1}$ refines exactly one event of $M_{i}$ (i.e., events cannot be merged);

5. each event in $M_{i+1}$ which refines an anticipated event of $M_{i}$ is itself either convergent or anticipated;

6. refinements of convergent or ordinary events of $M_{i}$ are ordinary in $M_{i+1}$.

7. no anticipated events remain in the final machine.

Figure 1 illustrates our development strategy for a vending machine, detailed in Sect. 3, where $C_{i}$ is the set of convergent events within $M_{i}$, and $O_{i}$ is the set of ordinary events within $M_{i}$. For example, $O_{0}$ $=\{$ selectItem, dispenseItem $\}$ and $C_{0}=\emptyset, O_{1}=\{$ selectBiscuit, selectChoc, dispenseBiscuit, dispenseChoc $\}$ and $C_{1}=\varnothing$ in $V M_{1}$. In $V M_{2}$ we note that $C_{2}=\{$ refund $\}$. In $V M_{3}$ we note that $C_{3}=\{$ refill $\}$ and in $V M_{4}$ we have $C_{4}=\{$ pay $\}$.

\section{Example}

In Sect. 2.3 we introduced a development strategy for a vending machine. Figures 3, 4, 5, 6 and 7 illustrate a development chain from machines $V M_{0}, V M_{1}, V M_{2}, V M_{3}$ to $V M_{4}$; there are no anticipated events in $V M_{4}$.

$V M_{0}$ is a simple machine that supports the selection and dispensing of items via two very simple events: selectItem and dispenseItem. The item variable is used to control when the events are enabled. VM decomposes the selection and dispensing to be specifically for chocolates and biscuits via four events: selectBiscuit, selectChoc, dispenseBiscuit and dispenseChoc. $V M_{1}$ contains a data refinement which tracks the item chosen rather simply counting the selection. The second refinement step introduces $V M_{2}$ and the notion of paying and refunding. The pay event in $V M_{2}$ is always enabled and allows positive credit to be input. The machine allows a biscuit to be chosen if it has not already been chosen and additionally provided a payment has been made; a chocolate selection is similar. Hence the guards of all four of the original events selectBiscuit, selectChoc, dispenseBiscuit and dispenseChoc are strengthened. The guard of the refund event means that credit cannot be refunded for selected items and cannot occur forever since it is convergent. Importantly, the refundEnabled flag is introduced so that it is only true after a dispense and prevents infinite loops of the pay followed by refund. 


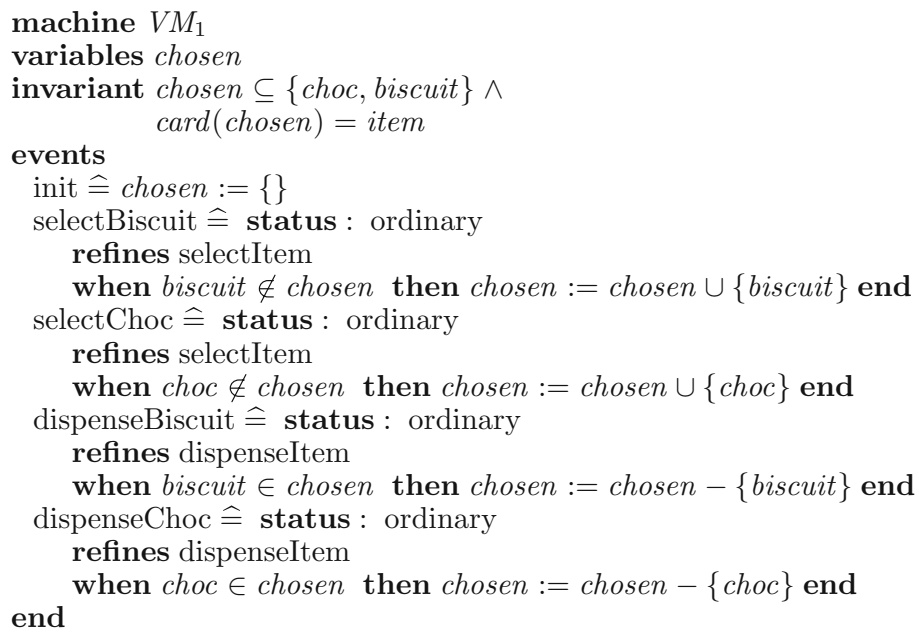

Fig. 4. $V M_{1}$

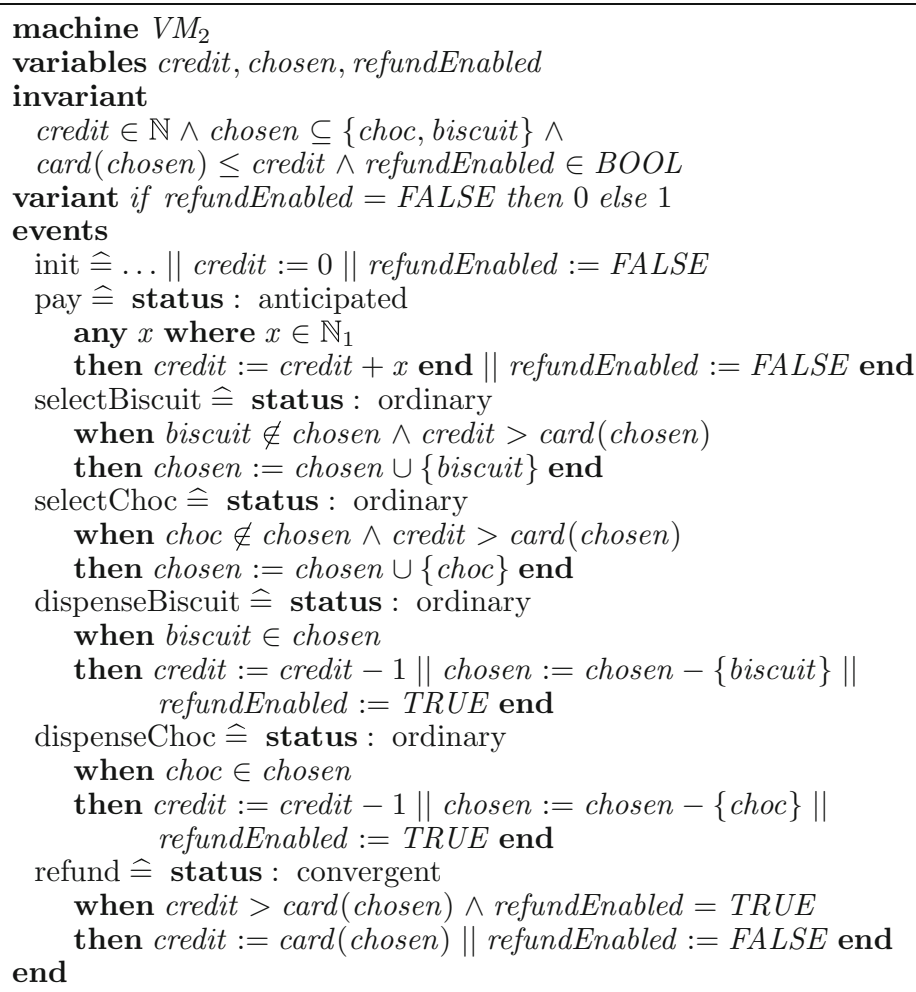

Fig. 5. $V M_{2}$ 


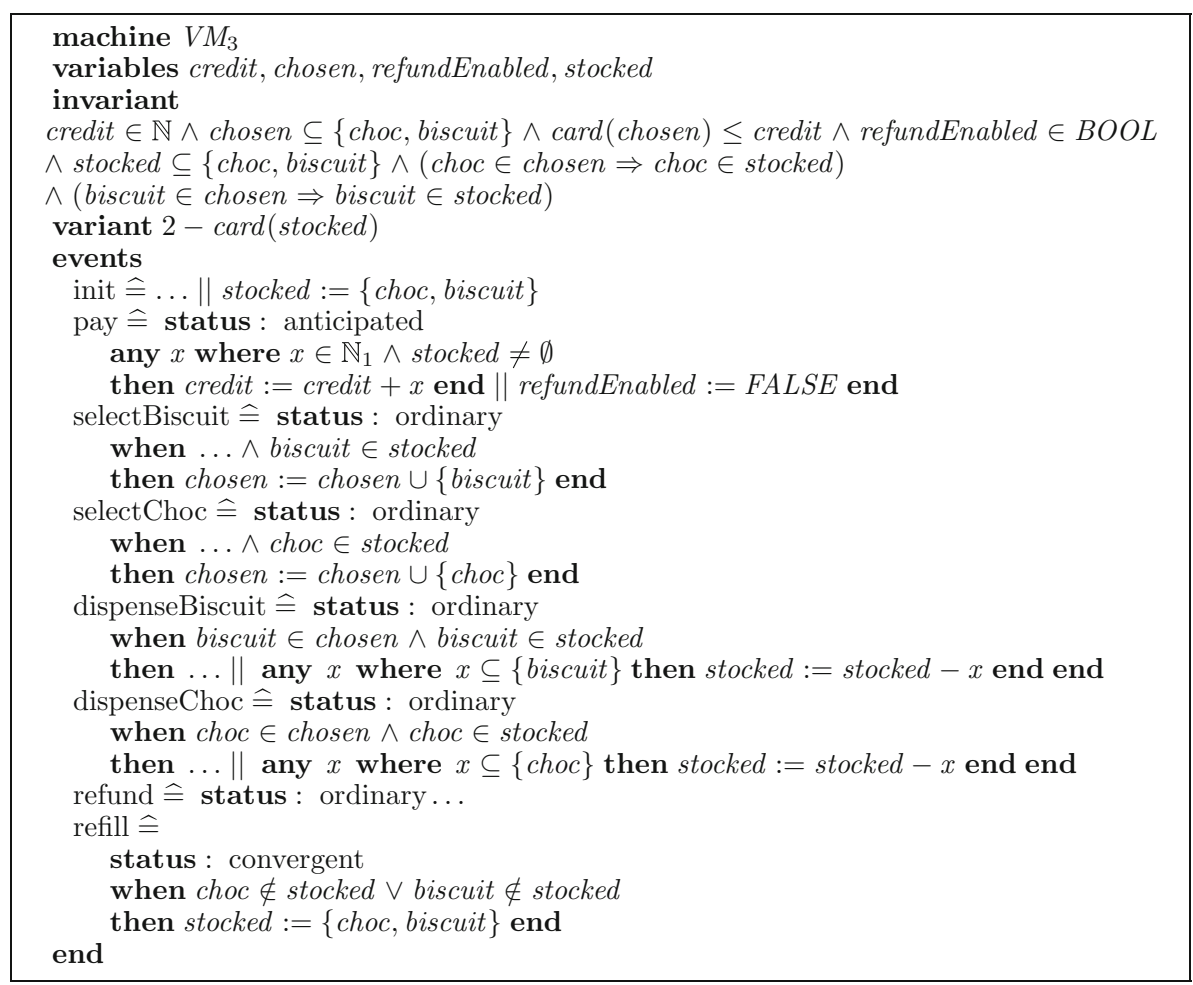

Fig. 6. $V M_{3}$

$V M_{3}$ introduces the notion of stocked items and a new refill event. We could have chosen many different guards for the refill event. For example, we could have labelled it anticipated with a guard of true. Instead we have made an underspecification where the stock can be restocked when there may be no biscuits or no chocolates, and established convergence. Again the guard of the four events introduced in $V M_{1}$ have been strengthened so that they are only enabled when the appropriate stocked item is in stock. But now dispenseBiscuit and dispenseChoc also capture the non-deterministic notion of running out or not of their respective items. The guard of refund remains unchanged. The guard of pay has been strengthened so that it is only enabled when there is stock but this is not strong enough to prevent it happening infinitely often, hence it remains anticipated in $V M_{3}$.

The final machine, $V M_{4}$, is a straightforward data refinement which introduces the capacity of the machine. Apart from highlighting the refinement relationship between stocked and chocStock and biscuitStock note the strengthening of the guard of refill so that vending machine should only be refilled when there is no stock. Also the guard of pay is strengthened so that it becomes convergent.

The example has been proved using Rodin 3.1.0 with Atelier-B 2.1.0. ${ }^{1}$ The development uses finite set variants instead of encodings in natural numbers to facilitate proof, for example the variant in $V M_{3}$ would be ITEM stocked. The proof statistics are as follows: context: 1,VM $: 6, V M_{1}: 18, V M_{2}: 19, V M_{3}: 9$ and $V M_{4}: 46$ proof obligations (44 automatic, 2 manual proofs). In total, there are 99 proof obligations (97 automatic).

\footnotetext{
${ }^{1}$ http://www.computing.surrey.ac.uk/personal/st/H.Treharne/papers/2015/VendingMachineDevelopment_FACS2015.zip.
} 


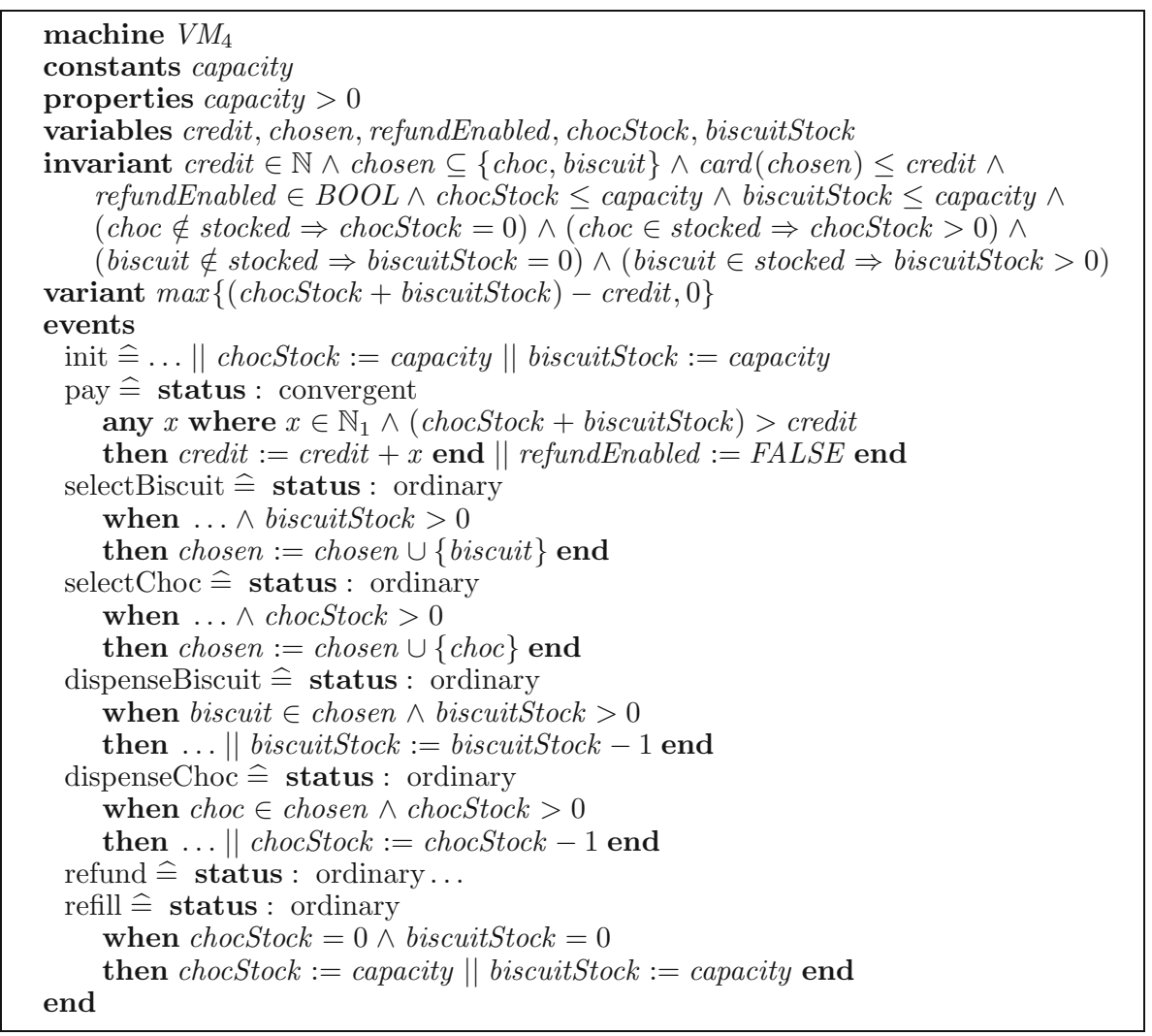

Fig. 7. $V M_{4}$

\section{Event-B semantics}

For a particular machine $M$ we define the ternary relation $T$ as follows: $\left(s, t, s^{\prime}\right) \in T \Leftrightarrow \exists x . G_{t}(x, s) \wedge$ $B A_{t}\left(s, x, s^{\prime}\right)$. This gives the relationship between the before and after state for the events within that machine where $s$ ans $s^{\prime}$ are states and $t$ is an event.

In this paper we define a trace (path) $u$ of $M$ as a finite or infinite sequence of alternating states and events (a,c or o), of the form, $\left\langle s_{0}, t_{0}, s_{1}, t_{1}, \ldots\right\rangle$ where $\forall i \geq 0 .\left(s_{i}, t_{i}, s_{i+1}\right) \in T$. A path $\left\langle s_{0}, t_{0}, \ldots, s_{k-1}, t_{k-1}, s_{k}\right\rangle$ is finite when $s_{k}$ is a deadlock state, i.e., $\neg \bigvee_{e \in \alpha M} G_{e}$ : none of the events of the machine are enabled. When a machine $M$ is deadlock free all of its traces are infinite.

Our definition of path is akin to Definition 2 in [PL10] where paths are also defined in terms of a ternary relation between states. Note that in [STWW14a] we did not need to consider the intermediate states as all our reasoning was based on the events of infinite traces. In this paper we need to consider intermediate states since we will be reasoning about LTL properties that require us to refer to the intermediate states.

We use the functions of projection $(\uparrow)$, length $(\#)$ and concatenation $(\frown)$ on finite and infinite sequences. Projection $u \uparrow E$ of a path $u$ onto a set of events $E$ gives the subsequence of transitions of $u$ that are in $E$. This is used for example in Definition 4.1 below. Note that $u\left\lceil\alpha M=\left\langle t_{0}, t_{1}, \ldots\right\rangle\right.$ represents the trace of all events in the path $u$. The length of $u, \#(u)=\#\left(u\lceil\alpha M)\right.$, is defined as the number of transitions in a path. ${ }^{2}$ A finite sequence of state transition pairs ending with an event can be concatenated with a path, i.e., $\left\langle s_{0}, t_{0}, \ldots t_{k}\right\rangle \frown u$. In this paper concatenation is simply used to partition a finite or infinite sequence of events.

\footnotetext{
${ }^{2}$ Note that length of a path in [PL10] is defined as the number of states in a path, however for this paper our focus is on events.
} 
We additionally need to define the relationship between abstract and concrete paths since we are interested in their relationship through refinement. Thus, in the following definition we relate abstract and concrete paths. Relation $R$ is defined as follows:

Definition 4.1 The relation $R$ is the weakest relation such that: $\left\langle s_{0}, t_{0}, s_{1}, t_{1}, \ldots\right\rangle$ is a path of $M_{i}$ and $\left\langle s_{0}^{\prime}, t_{0}^{\prime}, s_{1}^{\prime}, t_{1}^{\prime}, \ldots\right\rangle$ is a path of $M_{j}$ and $i<j$ and $\left\langle s_{0}, t_{0}, s_{1}, t_{1}, \ldots\right\rangle R\left\langle s_{0}^{\prime}, t_{0}^{\prime}, s_{1}^{\prime}, t_{1}^{\prime}, \ldots\right\rangle$ iff

- $\left\langle s_{0}^{\prime}, t_{0}^{\prime}, s_{1}^{\prime}, t_{1}^{\prime}, \ldots\right\rangle \uparrow \operatorname{dom}\left(g_{i+1, j}\right)$ is infinite

- if $t_{0}^{\prime} \in \operatorname{dom}\left(g_{i+1, j}\right)$ then $t_{0}=g_{i+1, j}\left(t_{0}^{\prime}\right) \wedge \mathcal{J}\left(s_{0}, s_{0}^{\prime}\right) \wedge \mathcal{J}\left(s_{1}, s_{1}^{\prime}\right) \wedge\left\langle s_{1}, t_{1}, \ldots\right\rangle R\left\langle s_{1}^{\prime}, t_{1}^{\prime}, \ldots\right\rangle$

- if $t_{0}^{\prime} \notin \operatorname{dom}\left(g_{i+1, j}\right)$ then $J\left(s_{0}, s_{0}^{\prime}\right) \wedge \mathcal{J}\left(s_{0}, s_{1}^{\prime}\right) \wedge\left\langle s_{0}, t_{0}, s_{1}, t_{1}, \ldots\right\rangle R\left\langle s_{1}^{\prime}, t_{1}^{\prime}, \ldots\right\rangle$

Note that the states are matched through $\mathcal{J}$ and $R$ must be the weakest relation in order to rule out false as a possible $R$. The relation is general to be between two paths and two arbitrary different refinement levels. The relation is not restricted to relating two adjacent refinement steps. This definition bears some resemblance to Definition 11 of [DJK03], though that paper is not concerned with the translation of LTL properties.

Note that the relation between two paths $u_{1}$ and $u_{2}$ (i.e., $u_{1} R u_{2}$ ) only holds if $u_{2}$ projected onto the alphabet of the events $u_{1}$ lives in is infinite. This means that all the events in the infinite trace $u_{1}$ will correspond to something in $u_{2}$. Consider an infinite trace $u_{1}$ related to an infinite trace $u_{2}$ which only had finitely many events in $u_{1}$ that it matched. For example, if $u_{1}=\langle a, a, a, a, \ldots\rangle$ and $u_{2}=\langle b, c, b, c, b, c, c, c, c, c, c, \ldots\rangle$ where $b$ maps to $a$ in the mapping function $g$, and $c$ is a new event. Then $R$ does not hold between these two paths, since the infinite condition on $u_{2}$ is not met. Our theoretical results require $R$ to be defined with respect to infinite traces since we will only need to consider infinite traces. We never need to consider finite abstract traces in our proofs, hence this definition is only required to define $R$ on infinite traces.

For example, a path of $V M_{0}$ is

$u_{0}=\langle 0$, selectItem, 1, selectItem, 2 , dispenseItem, 1 , selectItem $, 2, \ldots\rangle$

where the state $i$ carries the value of $i$ tem.

Similarly, a possible path of $V M_{1}$ is

$$
\begin{array}{r}
u_{1}=\langle\emptyset, \text { selectBiscuit },\{\text { biscuit }\}, \text { selectChoc, }\{\text { biscuit }, \text { choc }\}, \\
\text { dispenseChoc, }\{\text { biscuit }\}, \text { selectChoc, }\{\text { biscuit }, \text { choc }\}, \ldots\rangle
\end{array}
$$

where the state $s$ carries the value of chosen.

Then we have that $u_{0} R u_{1}$. We have that abstract states and concrete states are related by the linking invariant of $V M_{1}$, for example the fourth abstract state item $=1$ relates to the fourth concrete state chosen $=\{$ biscuit $\}$. We also have that the abstract and concrete events are mapped through $g_{1,1}$, for example the fourth concrete event is selectChoc and the fourth abstract event is selectItem. Since all the states are related through the linking invariant, and all the concrete events map to the abstract events, we have that $u_{0} R u_{1}$.

Now consider the following path of $V M_{2}$ :

$$
\begin{array}{cl}
u_{2}=\langle(0, \emptyset, \text { FALSE }), & \\
\text { pay, } & (3, \emptyset, F A L S E), \\
\text { selectBiscuit, } & (3,\{\text { biscuit }\}, F A L S E) \\
\text { selectChoc, } & (3,\{\text { biscuit, choc }\}, F A L S E), \\
\text { dispenseChoc, } & (2,\{\text { biscuit }\}, T R U E) \\
\text { refund, } & (1,\{\text { biscuit }\}, F A L S E), \\
\text { pay, } & (5,\{\text { biscuit }\}, F A L S E), \\
\text { selectChoc, }, & (5,\{\text { biscuit }, \text { choc }\}, F A L S E), \\
& \ldots
\end{array}
$$


Then $u_{1} R u_{2}$ : transitions for the new events pay and refund meet the third condition of Definition 4.1, and those for the events already present, e.g., selectBiscuit, meet the second condition. Hence it also follows that $u_{0} R u_{2}$ through two refinement steps.

Conversely, consider the path $u_{2}^{\prime}$ of $V M_{2}$ consisting of an infinite sequence of pay transitions:

$u_{2}^{\prime}=\langle(0, \emptyset, F A L S E)$, pay,$(1, \emptyset, F A L S E)$, pay $,(2, \emptyset, F A L S E), \ldots\rangle$

Such a path fails the first condition of Definition 4.1, since there are not infinitely many transitions corresponding to abstract events. Hence $u_{2}^{\prime}$ does not relate under $R$ to any path of $V M_{1}$ or $V M_{0}$.

Thus $R$ only related infinite abstract paths to infinite concrete paths.

A more complex behavioural semantics for B machines was given by Schneider et al. in [STW14] based on the weakest precondition semantics of [Mor90, But92] for action systems and CSP. In [STW14] there are two key results that enable us to reason about infinite sequences of convergent and ordinary events in this paper. Firstly, the following predicate captures that if an infinite trace $u$ performs infinitely many events from $C$ then it has infinitely many events from $O$, where $C$ and $O$ are sets of events.

Definition 4.2 $C A(C, O)(u) \widehat{=}(\#(u\lceil C)=\infty \Rightarrow \#(u\lceil O)=\infty)$

$C$ and $O$ will be used to capture convergent and ordinary events through a development. For an Event-B machine $M$ the above means that it does not diverge on its $C$ events. This is precisely what we get when we prove WFD_REF but the above definition describes the result on traces.

The second result from [STW14], restated as Theorem 4.3, allows us to conclude that there are no infinite sequences of convergent events in the final machine of a refinement chain $M_{n}$.

Theorem 4.3 If $M_{0} \preccurlyeq W M_{1} \preccurlyeq W \cdots \preccurlyeq W M_{n}$ then

$$
M_{n} \text { sat } C A\left(g_{1, n}^{-1}\left(C_{0}\right) \cup \cdots \cup g_{i, n}^{-1}\left(C_{i-1}\right) \cup \cdots \cup C_{n}, g_{1, n}^{-1}\left(O_{0}\right)\right)
$$

$M$ sat $S$ means that predicate $S$ holds for every infinite trace of machine $M$. The result for our example is

$V M_{4}$ sat $C A(\{$ pay, refund, refill $\}$, \{selectBiscuit, selectChoc, dispenseBiscuit, dispenseChoc $\left.\}\right)$

Thus $C A(\{$ pay, refund, refill\}, \{selectBiscuit, selectChoc, dispenseBiscuit, dispenseChoc $\})$ holds for all traces of $V M_{4}$. If there are infinitely many pay, refund and refill events then there must be inifinitely many select and dispense events. In other words an execution cannot reach a point where it only does pay, refund and refill events from then on. It must always progress to a select or dispense event.

Note that in particular the $C A$ property does not hold for the infinite trace $\langle p a y, p a y, p a y, \ldots\rangle$ therefore that trace cannot be a trace of $V M_{4}$. Indeed, we can see that $V M_{4}$ only allows pay while the amount of credit is less than the amount of stock held by the machine.

\section{LTL notation}

In this paper we use the following grammar for the LTL operators:

$$
\phi::=\text { true }|[t]| \neg \phi\left|\phi_{1} \vee \phi_{2}\right| \phi_{1} U \phi_{2}|p| X \phi \mid e(t)
$$

These operators differ from those presented in [STWW14a] since we have included the state predicate $p$, the next operator $X$ and the enabledness of an event $t, e(t)$. Note that we do not provide theoretical results for $e(t)$ until Sect. 7 since the theoretical results require a more detailed treatment of Event-B refinement than those for the other operators. It is the same grammar as presented by Plagge and Leuschel in [PL10] and hence supported by the ProB tool. 
A machine $M$ satisfies $\phi$, denoted $M \models \phi$, if all paths of $M$ starting from one its initial states satisfy $\phi$. The definition for $u$ to satisfy $\phi$ is defined by induction over $\phi$ as follows:

\section{Definition 5.1}

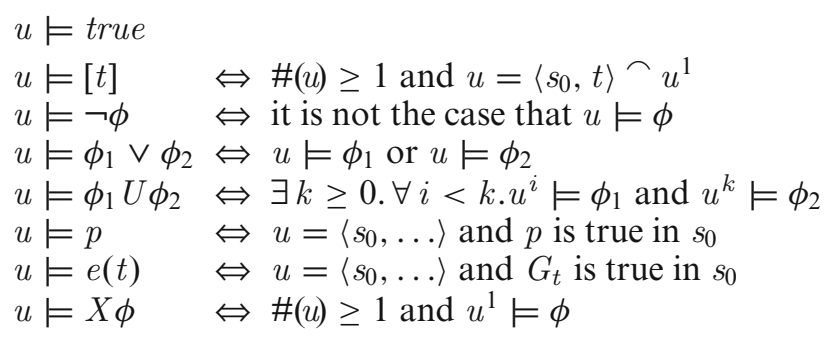

where $u^{n}$ is $u$ with the first $n$ state-transition pairs removed, i.e., $u=\left\langle s_{0}, t_{0}, \ldots, s_{n-1}, t_{n-1}\right\rangle \frown u^{n}$. The until operator is a strong until since there is a finite $k$ for which $u^{k} \models \phi_{2}$. Note that " $G_{t}$ is true in $s_{0}$ " is written as $G_{t}\left(s_{0}\right)$ in the proofs in this paper.

From these operators Plagge and Leuschel derived several additional operators, including: conjunction $\left(\phi_{1} \wedge\right.$ $\phi_{2}$ ), finally (or eventually) $(F \phi)$, and globally (or always) $(G \phi)$, in the usual way. From their definition of $F \phi=$ true $U \phi$ and $U$ from above it follows that $F([a] U[b])=F([b])$.

We also use these additional operators, and for explicitness we also provide direct definitions for them:

$$
\begin{array}{ll}
u=\phi_{1} \wedge \phi_{2} & \Leftrightarrow u \models \phi_{1} \text { and } u \models \phi_{2} \\
u=F \phi & \Leftrightarrow \exists i \geq 0 . u^{i} \models \phi \\
u \models G \phi & \Leftrightarrow \forall i \geq 0 . u^{i} \models \phi
\end{array}
$$

For example, the informal specification for the Lift given in Sect. 1, that whenever top happens then eventually ground will happen, could be written as $G([t o p] \Rightarrow F[$ ground $])$. Similarly, for our running example given in Sect. 3, we can state that whenever selectItem happens then eventually dispenseItem will happen is captured using the following LTL property. We reference it for use later in the paper.

$$
\phi_{A}=G([\text { selectItem }] \Rightarrow F[\text { dispenseItem }])
$$

From our running $V M$ example, in [STWW14b] we predominantly discussed LTL properties of the form the predicate $G F$ [selectBiscuit] which expresses that selectBiscuit occurs infinitely often: at any point there is always some occurrence of selectBiscuit at some point in the future. We used this construction to express properties such as:

$$
\phi_{B}=(\neg G F[\text { selectBiscuit }]) \Rightarrow G([\text { selectChoc }] \Rightarrow F[\text { dispenseChoc }])
$$

This states that provided selectBiscuit only occurs finitely often (i.e., eventually stops), then whenever selectChoc occurs then dispenseChoc will eventually occur.

In [STWW14b] we also discussed how LTL properties of an abstract machine become transformed through a refinement step. For example, one LTL property of $V M_{0}$ is $G F[$ selectItem] which states that from any state that is reached, selectItem will eventually occur. This translates to the property $G F([$ selectBiscuit $] \vee[$ selectChoc $]$ ) for $V M_{1}$ and the property remains unchanged through the remaining refinement steps.

Appropriate properties for $V M_{0}$ in this paper are:

$$
\begin{aligned}
\phi_{C} & =G(\text { item }=2 \Rightarrow(X(\text { item }=1))) \\
\phi_{D} & =G(\text { item }=0 \Rightarrow(X(\text { item }=1)))
\end{aligned}
$$

Notice that $\phi_{C}$ and $\phi_{D}$ use the next and proposition operator which are new to this paper's results. In Section 6 we will discuss how these are transformed and preserved through refinement. Property $\phi_{C}$ states that when you select the maximum amount of items then the amount of selected items will be decreased in the next state. This is true since the dispense item is the only event that can happen when item $=2$ and this is true in every state where the maximum number of items have been chosen. Property $\phi_{D}$ is similar in style to $\phi_{C}$ and states that whenever item is 0 then the only thing that can happen is that an item will be selected and hence in the next state the value of item will be 1 . 
It will also be useful to identify the events mentioned explicitly in an LTL formula $\phi$. This set is called the alphabet of $\phi$. This is written $\alpha(\phi)$, similar to the use of $\alpha M$ for the alphabet of machine $M$. For LTL formulae it is defined inductively as follows:

\section{Definition 5.2}

$$
\begin{aligned}
\alpha(\text { true }) & =\emptyset \\
\alpha([t]) & =\{t\} \\
\alpha(\neg \phi) & =\alpha(\phi) \\
\alpha\left(\phi_{1} \vee \phi_{2}\right) & =\alpha\left(\phi_{1}\right) \cup \alpha\left(\phi_{2}\right) \\
\alpha\left(\phi_{1} \wedge \phi_{2}\right) & =\alpha\left(\phi_{1}\right) \cup \alpha\left(\phi_{2}\right) \\
\alpha\left(\phi_{1} U \phi_{2}\right) & =\alpha\left(\phi_{1}\right) \cup \alpha\left(\phi_{2}\right) \\
\alpha(F \phi) & =\alpha(\phi) \\
\alpha(G \phi) & =\alpha(\phi) \\
\alpha(p) & =\emptyset \\
\alpha(e(t)) & =\{t\} \\
\alpha(X \phi) & =\alpha(\phi)
\end{aligned}
$$

For example, we have $\alpha\left(\phi_{A}\right)=\{$ selecItem, dispenseItem $\}$ and $\alpha\left(\phi_{C}\right)=\alpha\left(\phi_{D}\right)=\emptyset$ for $\phi_{A}, \phi_{C}$ and $\phi_{D}$ above.

\section{Preserving LTL properties}

In this section we provide results to demonstrate when properties are preserved by refinement chains of the form: $M_{0} \preccurlyeq W M_{1} \preccurlyeq_{W} \cdots \preccurlyeq{ }_{W} M_{n}$. The results are general in order to deal with splitting events in Event-B, which occurs when abstract events are refined by several events in the concrete machine, corresponding to a set of alternatives.

Recall that our running example contains an example of splitting where the events in $V M_{0}$ are each refined by two events: selectItem is refined by both selectBiscuit and selectChoc, and dispenseItem is refined by both dispenseBiscuit and dispenseChoc. Recall that a renaming function $f$ associated concrete events with abstract events that they refine. For example $f_{1}($ selectChoc $)=$ selectItem and $f_{1}($ selectBiscuit $)=$ selectItem where $f_{1}$ is the renaming function appropriate for mappings between $V M_{1}$ and $V M_{0}$. In Sect. 2 we defined a compositional mapping $g_{i, j}$ in terms of the individual $f$ mappings. Thus, for the chain $V M_{0} \preccurlyeq W V M_{1} \preccurlyeq W \cdots \preccurlyeq{ }_{W} V M_{4}$, we obtain that $g_{1,4}($ selectBiscuit $)=g_{1,4}($ selectChoc $)=$ selectItem, and $g_{1,4}$ (dispenseBiscuit $)=g_{1,4}($ dispenseChoc $)=$ dispenseItem, and $g_{1,4}$ is not defined on the remaining events of $V M_{4}$.

This section excludes a discussion of temporal properties that use the enabled operator, since these require the more elaborate treatment of Sect. 7.

We begin with a LTL property that will hold for all refinement chains that observe the strategy from Sect. 2.3. It is restated from [STWW14b].

\subsection{General GF property preservation}

Lemma 6.1 states that the final machine in the refinement chain must always eventually perform some event relating to an event in the initial machine. In other words, $M_{n}$ will perform infinitely many of the initial events. This means that the events introduced along the refinement chain cannot occur forever at the expense of the original events. In our example, $\alpha M_{0}=O_{0}$.

Lemma 6.1 If $M_{0} \preccurlyeq W \quad M_{1} \preccurlyeq W \cdots \preccurlyeq W \quad M_{n}$ and $M_{n}$ is deadlock free and $M_{n}$ does not contain any anticipated events then $M_{n} \models G F\left(\bigvee_{t \in g_{1, n}^{-1}\left(\alpha M_{0}\right)}[t]\right)$

Observe that if there is no renaming or splitting, then $g_{1, n}$ is the identity function on the events in $\alpha M_{0}$, and the disjunction simplifies to be the disjunction of the events in the alphabet of $M_{0}$. 


\subsection{General LTL property preservation (excluding the enabled operator)}

The new results in this paper are given in Lemmas 6.3 and 6.4. They concern the preservation of temporal properties that could be introduced at any refinement step and are required to be preserved by subsequent refinement steps. We will see that Lemmas 6.3 and 6.4 are variants of each other. The two subtly different lemmas are needed due to the two ways of dealing with the negation operator in the associated proofs.

We have already observed from the vending machine example that events can be split as shown above and that new events can be introduced during a refinement, e.g., pay, refill, in $V M_{2}$ and $V M_{3}$. We aim for LTL properties to hold even though splitting of events may occur and new anticipated and convergent events are being introduced. i.e., we aim for a result that states when a property is introduced in refinement step $i$ then the property must hold for machine $M_{i}$ and the transformed property also holds at the end of the refinement chain at level $n$ for machine $M_{n}$.

To aid the formalisation of the lemmas we introduce Definition 6.2 to define a translation function trans $_{g}$ with respect to renaming function $g$ as mapping LTL predicates on abstract events to predicates on their corresponding concrete events as follows:

Definition 6.2 Let $g \in B \rightarrow A$ and define $N=B-\operatorname{dom}(g)$

$$
\begin{aligned}
\operatorname{trans}_{g}(\operatorname{true}) & =\operatorname{true} \\
\operatorname{trans}_{g}([t]) & =\bigvee_{n \in N}[n] U \bigvee_{y \mid g(y)=t}[y] \\
\operatorname{trans}_{g}(\neg \phi) & =\neg \operatorname{trans}_{g}(\phi) \\
\operatorname{trans}_{g}\left(\phi_{1} \vee \phi_{2}\right) & =\operatorname{trans}_{g}\left(\phi_{1}\right) \vee \operatorname{trans}_{g}\left(\phi_{2}\right) \\
\operatorname{trans}_{g}\left(\phi_{1} \wedge \phi_{2}\right) & =\operatorname{trans}_{g}\left(\phi_{1}\right) \wedge \operatorname{trans}_{g}\left(\phi_{2}\right) \\
\operatorname{trans}_{g}\left(\phi_{1} U \phi_{2}\right) & =\operatorname{trans}_{g}\left(\phi_{1}\right) U \operatorname{trans}_{g}\left(\phi_{2}\right) \\
\operatorname{trans}_{g}(G \phi) & =G \operatorname{trans}_{g}(\phi) \\
\operatorname{trans}_{g}(F \phi) & =F \operatorname{trans}_{g}(\phi) \\
\operatorname{trans}_{g}(p) & =\exists s . \mathcal{J}\left(s, s^{\prime}\right) \wedge p \text { is true in } s \\
\operatorname{trans}_{g}(X \phi) & =\bigvee_{n \in N}[n] U\left(\underset{d \in \operatorname{dom}(g)}{\bigvee}[d] \wedge X\left(\operatorname{trans}_{g}(\phi)\right)\right)
\end{aligned}
$$

The translation is with respect to $g$ since it can be used over a number of refinement steps. When we apply the definition, $B$ and $A$ will be particular alphabets of machines and $\mathcal{J}$ will be the composition of the linking invariant between the two machines. This definition allows us to take into account the new events that are introduced during refinement steps as required in clauses (6) and (14). It is inspired by [WdRF12] and we will reflect on this in Sect. 8.

Clause (6) translates the occurrence of the abstract event $t$ to the (repeated) occurrences of the new events introduced during the refinement $\left(\bigvee_{n \in N}[n]\right)$ until the occurrence of one of the concrete events that maps to the abstract event $t$. Note that the new events cannot happen forever since it is a strong until operator. Clause (14) translates $X \phi$ to the occurrences of new events until one of the concrete events which do relate to an abstract event occurs, and translation of the property holds in the next state $\left(X\left(\operatorname{trans}_{g}(\phi)\right)\right)$.

Clause (13) translates an abstract predicate $p$ on states to a concrete predicate on states that relate to the abstract ones.

For example, consider $B=\alpha\left(V M_{1}\right)$ and $A=\alpha\left(V M_{0}\right)$. In this case $g=f_{1}$ and $N=\emptyset$. Hence,

$$
\operatorname{trans}_{f_{1}}\left(\phi_{A}\right)=G(([\text { selectBiscuit }] \vee[\text { selectChoc }]) \Rightarrow F([\text { dispenseBiscuit }] \vee[\text { dispenseChoc }]))
$$

as trans $_{f_{1}}([$ selectItem $])=[$ selectBiscuit $] \vee[$ selectChoc $]$. Since there are no new events in $V M_{1}$ the expression containing the until operator simplifies to the disjunction of the corresponding mapped events. Similarly the translation of the occurrence dispenseItem event translates to the disjunction of the concrete dispense events. 
The same $\phi_{A}$ property translates for a refinement chain as follows: consider $B=\alpha\left(V M_{2}\right)$ and $A=\alpha\left(V M_{0}\right)$. In this case $g=f_{2} ; f_{1}$ and $N=\{$ pay, refund $\}$. There are new events in $V M_{2}$ which have an impact on $\phi_{A}$. Hence,

$$
\begin{aligned}
\text { trans }_{f_{2} ; f_{1}}\left(\phi_{A}\right) & =G\left(\left(\bigvee_{n \in N}[n] U([\text { selectBiscuit }] \vee[\text { selectChoc }])\right)\right. \\
& \Rightarrow F([\text { dispenseBiscuit }] \vee[\text { dispenseChoc }]))
\end{aligned}
$$

It states that new events from $V M_{2}$ are permitted to occur until one of the mapped selection events happens the new events are again permitted to occur until one of the concrete dispensing events occurs. Notice that the eventually property is simplified from $F\left(\left(\bigvee_{n \in N}\right)[n] U([\right.$ dispenseBiscuit $] \vee[$ dispenseChoc $\left.])\right)$ since $F([a] U[b])=F([b])$ as noted in Sect. 5.

Using the same simple refinement chain from $V M_{2}$ to $V M_{0}$ an example to illustrate the translation of Clause (13) for property $\phi_{C}$ is given as follows:

$$
\text { trans }_{g}\left(\phi_{C}\right)=G\left(\text { trans }_{g}(\text { item }=2) \Rightarrow \text { trans }_{g}(X(\text { item }=1))\right)
$$

The predicate trans $_{g}($ item $=2)$ becomes $\exists$ item. $(\#($ chosen $)=$ item $\wedge$ item $=2)$ which simplifies to $\#($ chosen $)=2$. Also, trans $_{g}(X($ item $=1))$ simplifies to

$$
\begin{aligned}
& ([\text { pay }] \vee[\text { refund }]) \\
& \quad U([\text { dispenseBiscuit }] \vee[\text { dispenseChoc }] \vee[\text { selectBiscuit }] \vee[\text { selectChoc }]) \wedge X(\#(\text { chosen })=1)
\end{aligned}
$$

In a state where $\#($ chosen $)=2$, both the dispenseBisuit and dispenseChoc concrete events are possible and also one of the new events in $N$, namely pay. Hence, through the translation of $\operatorname{trans}_{g}(X($ item $=1))$ any number of pay events can occur until one of the dispense events occurs and then in the next state we assert there is a matching concrete state when item $=1$ holds. There will be a matching concrete state because one of the dispense events will have occurred, i.e., the concrete state of chosen will have decreased the number of its elements by one and therefore the linking invariant will hold.

Two main results are presented in this section which identify additional conditions under which an LTL property $\phi$ will be preserved in a refinement chain that adheres to the development strategy given in Sect. 2.3. The strategy already makes clear that by the end of the refinement chain there should be no outstanding anticipated events (and so all newly introduced events have been shown to be convergent), restriction 6 of the Development Strategy of Sect. 2.3.

The common additional conditions for both new results are as follows:

Condition $1 \phi$ must not contain the enabled (e) operator.

Condition 2 the final machine in the refinement chain must be deadlock-free.

These conditions are enough to ensure that $\phi$ is preserved through refinement chains in the case where the LTL also does not contain the negation $(\neg)$ operator. In particular, if a property $\phi$ is established for $M_{i-1}$, then the resulting system $M_{n}$ will satisfy the translation of the property: $\operatorname{trans}_{g_{i, n}}(\phi)$. Hence, by using Definition 6.2 with $g=g_{i, n}$ we are now able to define the first result: Lemma 6.3.

Lemma 6.3 If $M_{i} \models \phi$ and $\phi$ does not contain the enabled (e) and negation $(\neg)$ operators and $M_{i} \preccurlyeq W \cdots \preccurlyeq W M_{n}$ and $0 \leq i<n, M_{n}$ is deadlock free and $M_{n}$ does not contain any anticipated events then $M_{n} \models$ trans $_{g_{i+1, n}}(\phi)$

Note the requirement for the refinement chain to adhere to restriction 6 of the Development Strategy of Sect. 2.3 we mention this explicitly in the lemma to emphasise it. The second lemma, Lemma 6.4, is similar but subtly different; in order to have a result on the negation operator we need to restrict the composition of the refinement relations $\mathcal{J}$ to be functional. This same restriction was identified by Derrick and Smith in [DS12]. Hence a further condition is needed for this result as follows:

\section{Condition 3}

The compositional linking invariant $\mathcal{J}$ must be functional. In other words, $\mathcal{J}\left(v_{1}, w\right) \wedge \mathcal{J}\left(v_{2}, w\right) \Rightarrow v_{1}=v_{2}$

Lemma 6.4 If $M_{i} \models \phi$ and $\phi$ does not contain enabled (e) operator and $M_{i} \preccurlyeq W \cdots \preccurlyeq W M_{n}$ and $0 \leq i<n$, the composition of the refinement relations $\mathcal{J}$ is functional and $M_{n}$ is deadlock free and $M_{n}$ does not contain any anticipated events then $M_{n} \models$ trans $_{g_{i+1, n}}(\phi)$ 
Note that unlike Lemma 6.1 the application of this lemma requires a property to be established of $M_{i}$.

The proofs for the lemmas are given in Appendix A. In order to apply Lemmas 6.3 and 6.4 in practice Condition 1 can be achieved by a simple review and Condition 2 is a mechanical check that can be done automatically using ProB. Condition 3 must also achieved by review, but note that if each of the linking invariants is functional then so is their composition. The proof obligations related to the refinement chain would need to be proved using Rodin or AtelierB.

\subsection{Preserving vending machine properties}

The purpose of this section is to consider the LTL properties of interest for the vending machine development, to highlight that:

- properties need not hold through all refinement steps, only for the machine in the refinement step in which the properties are introduced and again for the machine at the end of the refinement chain which does not contain anticipated events.

- properties can be introduced at different levels of refinement, minimising the need for duplicate LTL properties to be introduced in subsequent refinement steps,

We consider the application of the above Lemmas to our running example on the refinement chain

$V M_{0} \preccurlyeq W \quad V M_{1} \preccurlyeq W \quad V M_{2} \preccurlyeq W \quad V M_{3} \preccurlyeq W \quad V M_{4}$

In this case we obtain immediately from Lemma 6.1 that

$$
V M_{4} \models G F([\text { selectBiscuit }] \vee[\text { selectChoc }] \vee[\text { dispenseBiscuit }] \vee[\text { dispenseChoc }])
$$

Any execution of $V M_{4}$ will involve infinitely many occurrences of some of these events. The newly introduced events pay, refund, refill cannot be performed forever without the occurrence of the original events.

We have already introduced four properties which we recap here as follows:

$$
\begin{aligned}
& \phi_{A}=G([\text { selectItem }] \Rightarrow F[\text { dispenseItem }]) \\
& \phi_{B}=(\neg G F[\text { selectBiscuit }]) \Rightarrow G([\text { selectChoc }] \Rightarrow F[\text { dispenseChoc }]) \\
& \phi_{C}=G(\text { item }=2 \Rightarrow(X(\text { item }=1))) \\
& \phi_{D}=G(\text { item }=0 \Rightarrow(X(\text { item }=1)))
\end{aligned}
$$

Note that property $\phi_{B}$, and properties $\phi_{G}, \phi_{H}$ and $\phi_{I}$ introduced below, were discussed in the example in [STWW14b] but we restate them here so that it is clear that the new results apply to them.

Properties $\phi_{A}, \phi_{C}$ and $\phi_{D}$ are appropriately introduced in the first development step upon specification of $V M_{0}$ since they relate to events in $V M_{0}$. We have checked that $V M_{0}$ satisfies each of these three properties using ProB. Once we have also established the refinement chain $V M_{0} \preccurlyeq{ }_{W} V M_{1} \preccurlyeq{ }_{W} V M_{2} \preccurlyeq{ }_{W} V M_{3} \preccurlyeq{ }_{W} V M_{4}$, and that $V M_{4}$ is deadlock free we can deduce using Lemma 6.3 that the translations of all three properties hold for $V M_{4}$.

For example, from the result for $V M_{0}$ that whenever selectItem occurs then dispenseItem will eventually occur, $V M_{0} \models \phi_{A}$, we obtain from Lemma 6.3 that

$$
V M_{4} \models G(([\text { selectBiscuit }] \vee[\text { selectChoc }]) \Rightarrow F([\text { dispenseBiscuit }] \vee[\text { dispenseChoc }]))
$$

This states that whenever selectBiscuit or selectChoc occur, then dispenseBiscuit or dispenseChoc will eventually occur.

Property $\phi_{B}$ is an appropriate property for first refinement step and $V M_{1} \models \phi_{B}$, which we have checked using ProB. Once again provided we establish the correctness of the refinement chain and demonstrate that $V M_{4}$ is deadlock free then we can deduce using Lemma 6.4 that $V M_{4} \models \operatorname{trans}_{g_{1,4}}\left(\phi_{C}\right)$. Property $\phi_{B}$ states that if you do not always eventually have a selectBiscuit then you will be able to choose a chocolate and for it to be dispensed. There is a dual property when you do not always have a selectChoc.

Observe however that Lemmas 6.3 and 6.4 do not establish that the translation of $\phi$ properties hold in all refinement machines, only those deadlock free and with no anticipated events. For example, $V M_{2}$ does not satisfy the translations of $\phi_{A}$ nor $\phi_{B}$ since pay is anticipated and can be executed infinitely often and hence $V M_{2} \not \forall \operatorname{trans}_{f_{2} ; f_{1}}\left(\phi_{A}\right)$. The same applies for $V M_{3}$ since pay also remains anticipated in this machine. 
There are well-known benefits to introducing a property as early as possible. Firstly, we can minimise duplication of properties. For example, we need not check the following property to be required of $V M_{1}$

$$
G(([\text { selectBiscuit }] \vee[\text { selectChoc }]) \Rightarrow F([\text { dispenseBiscuit }] \vee[\text { dispenseChoc }]))
$$

We have already sufficient proof that it is true since $V M_{0} \models \phi_{A}$ and by application of Lemma 6.3.

$V M_{0}$ has three states while $V M_{1}$ has more states and $V M_{4}$ has unboundedly many states. While model checking a machine with three states is trivial, the time taken to perform explicit state model checking grows exponentially with the size of the statespace and model checking an infinite statespace is intractable in this manner. Therefore, it is easier to check $\phi_{A}$ on $V M_{0}$ than on $V M_{1}$ and it is not possible to check it on $V M_{4}$. Hence, through the application of our theoretical results we can establish properties of infinite state systems using our development strategy within existing tools.

There may be properties that do not hold early in a refinement chain but do hold later. For example, consider the following property:

$$
\phi_{E}=G([\text { pay }] \Rightarrow F([\text { dispenseBiscuit }] \vee[\text { dispenseChoc }]))
$$

The infinite behaviour of pay means that $\phi_{E}$ is not satisfied in $V M_{2}$. Thus, even though $\phi_{E}$ satisfies Condition 1, Lemma 6.3 is not applicable in the second refinement step because Condition 2 is not satisfied. However, $V M_{4} \models \phi_{E}$ since pay is no longer anticipated, i.e., convergent in $V M_{4}$. Hence, the property is not satisfied until the final refinement step. Since $V M_{4}$ is infinite state we cannot check this using ProB directly. However, it can be deduced from the result $V M_{4} \models \phi_{F}$ below together with Lines 15 and 16 above.

There may be properties involving anticipated events which hold at an early point of the refinement chain, and hence by the applications of the lemmas also hold at the end of the refinement chain. For example,

$$
\phi_{F}=G([\text { pay }] \Rightarrow(G[\text { pay }] \vee F([\text { selectBiscuit }] \vee[\text { selectChoc }]))
$$

$V M_{2} \models \phi_{F}$ and so by application of Lemma $6.3 V M_{4}$ also satisfies the translation of $\phi_{F}$. Since pay is anticipated, it cannot occur forever in $V M_{4}$ (as indicated in Line 15), the disjunct $G[$ pay $]$ in $\phi_{F}$ cannot hold in executions of $V M_{4}$ and we obtain

$$
V M_{4} \models G([\text { pay }] \Rightarrow F([\text { selectBiscuit }] \vee[\text { selectChoc }])
$$

This combines with the result of Line 16 to yield the result that $V M_{4} \models \phi_{E}$. Hence even though $\phi_{E}$ could not be established for $V M_{4}$ by direct use of Lemma 6.3, it follows from other results which are established by that lemma and others.

\section{Extending LTL properties to include enabled events}

In this section we extend the theoretical results to support the inclusion of the enabled $(e(t))$ operator from our LTL grammar in Sect. 5. First we extend Definition 6.2 to include the following translation clause to the definition of trans $_{g}$ for the mapping the enabledness of an abstract event to the disjunction of its corresponding concrete events as follows:

Definition 7.1 Let $g \in B \rightarrow A$ and define $N=B-\operatorname{dom}(g)$

$$
\operatorname{trans}_{g}(e(t))=\bigvee_{n \in N}[n] U\left(\left(\bigwedge_{n \in N} \neg e(n) \wedge \bigvee_{y \mid g(y)=t} e(y)\right) \vee\left(\bigvee_{n \in N} e(n) \wedge \bigvee_{d \in \operatorname{dom}(g)}[d]\right)\right)
$$

This additional clause was inspired by the definition of availability in [WdRF12]. This translation clause means that the occurrence of one of the new events introduced during the refinement $\left(\bigvee_{n \in N}[n]\right)$ is permitted until either:

1. a stable state is reached (none of the guards of the new events are enabled) and one of the guards of the concrete events that maps to the abstract event $t$ is enabled, or

2. a concrete event in $\operatorname{dom}(g)$ that corresponds to an abstract event occurs from an unstable state (where some of the new events are enabled). 

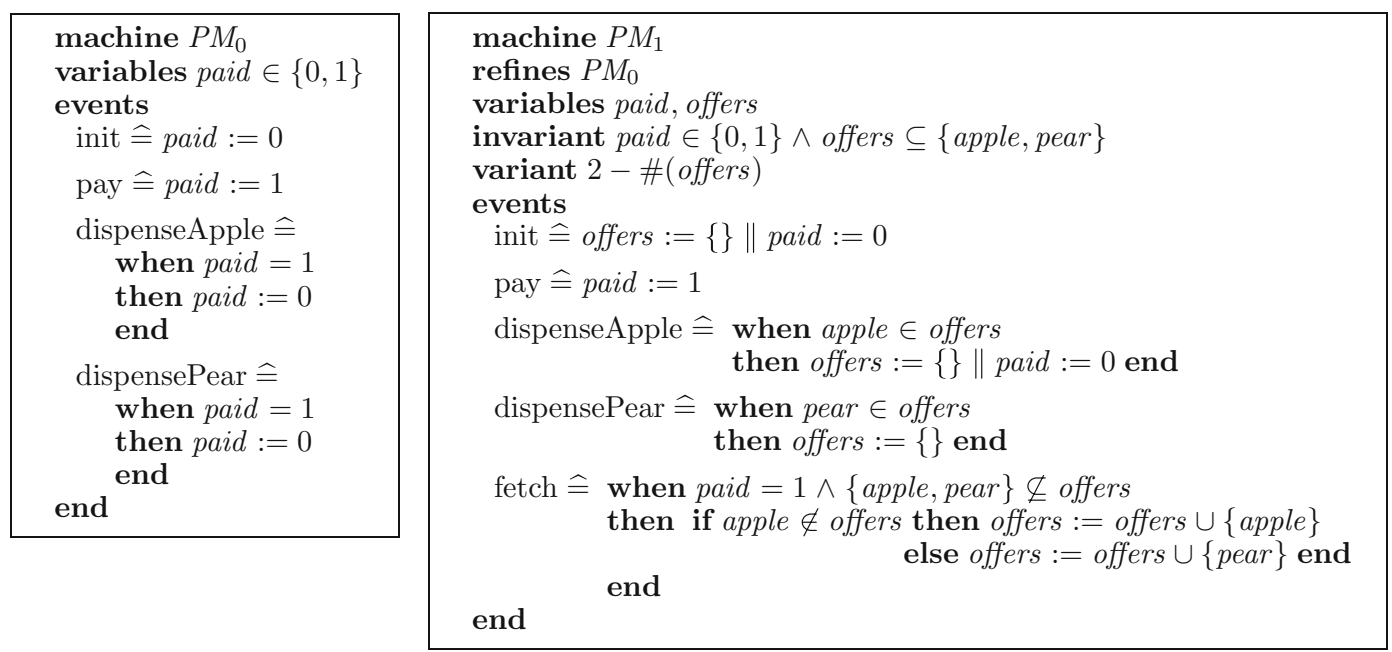

Fig. 8. Machine $P M_{0}$ and its refinement $P M_{1}$

To motivate this definition, consider the machines $P M_{0}$ and $P M_{1}$ of Fig. 8. Here we see that the events dispenseApple and dispensePear are both enabled in $P M_{0}$ following a pay event, thus for example the predicate $e($ dispensePear $)$ holds following the sequence [pay], i.e., $P M_{0}=\phi_{G}$, where $\phi_{G}=G([$ pay $] \Rightarrow X($ e(dispensePear $)$ ). The refinement machine $P M_{1}$ maintains a set offers of pieces of fruit which are available, and also introduces a new event fetch which adds items to that set.

Consider the translation of $\phi_{G}$, in particular, that of e(dispensePear). Consider the clause $\left(\bigwedge_{n \in N} \neg e(n) \wedge\right.$ $\left.\bigvee_{y \mid g(y)=t} e(y)\right)$ of Definition 7.1 regarding reaching the stable state. For the translation of $e($ dispensePear $)$, this is simplified to $\neg e($ fetch $) \wedge e($ dispensePear $)$. Following the occurrence of pay, it is not immediately the case that e(dispensePear) holds for $P M_{1}$, but if a user of that machine allows fetch to occur until it is no longer possible, corresponding to the sequence of events [pay, fetch, fetch] then indeed $e($ dispensePear $)$ is true following that sequence. This corresponds to clause (1).

With respect to the clause $\left(\bigvee_{n \in N} e(n) \wedge \bigvee_{d \in \operatorname{dom}(g)}[d]\right)$ regarding unstable states, in our example becomes $e($ fetch $) \wedge([$ pay $] \vee[$ dispenseApple $] \vee[$ dispensePear $])$, consider the following: the introduction of the new event fetch means that there are some intermediate states in which dispensePear is not yet enabled while the sequence of fetch events is ongoing. In our example the event dispenseApple becomes enabled after a single fetch, and if a user selects that in preference to waiting for fetch to occur, corresponding to the sequence [pay, fetch, dispenseApple], then a different path has been taken and the offer of dispensePear is not made anywhere along this path. This illustrates that some other event might occur before the event of interest becomes enabled in the refinement machine. We only wish to allow for this situation of dispensePear not yet being enabled if some further new event is still enabled, hence the inclusion of $\bigvee_{n \in N} e(n)$ in clause (2). If no further new events are possible, then an event such as dispensePear enabled in the abstract machine should also be enabled in the refinement machine.

In general, observe that if there are no new events $N$, then $\bigvee_{n \in N}[n]=$ false, $\bigvee_{n \in N}(e(n))=$ false, $\bigwedge_{n \in N} \neg e(n)$ $=$ true, and hence trans $_{g}(e(t))$ simplifies in this case to $\bigvee_{y \mid g(y)=t} e(y)$

For example, in $V M_{1}$ there are no new events, so trans $_{f_{1}}($ (e(dispenseItem $)$ ) simplifies to e(dispenseBiscuit) $\vee$ e(dispenseChoc).

Now we can examine whether the previous results from Sect. 6 remain applicable if we permit the enabled operator. In fact it turns out that they are not applicable; we need to strengthen the antecedents of the lemmas. We illustrate this on Lemma 6.3, but with the enabled operator $e$ permitted.

Consider the new LTL property which is true of $V M_{4}$ :

$$
\left.\phi_{I}=G([\text { selectBiscuit }] \Rightarrow(\text { Xe(dispenseBiscuit }))\right)
$$

and the refinement chain $V M_{4} \preccurlyeq W V M_{5}$ where $V M_{5}$ is given in Fig. 9. It is exactly the same as $V M_{4}$ except that the availability of the dispenseBiscuit is less, since its guard is strengthened so that a biscuit can only be dispensed once a biscuit and a chocolate are chosen. 


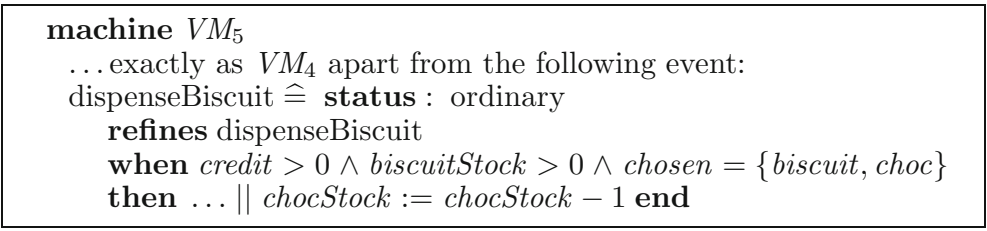

Fig. 9. $V M_{5}$

Hence, application of a relaxed Lemma 6.3 would state that $V M_{5}$ satisfies the trivial translation of $\phi_{I}$. However, this is not the case. For example, the infinite trace

$$
\langle(0, \emptyset, F A L S E), \text { pay, }(1, \emptyset, F A L S E), \text { pay, }(2, \emptyset, F A L S E), \text { selectBiscuit },(2,\{\text { biscuit }\}, F A L S E), \ldots\rangle
$$

does not satisfy trans $_{f_{5}}\left(\phi_{I}\right)=\phi_{I}$ since dispenseBiscuit is not enabled in the fourth state; selectChoc has not yet occurred to increase the value of the set chosen appropriately.

This example illustrates that we need a stronger notion of refinement that deals with the liveness of events so that we can ensure that the enabledness of events is guaranteed through the refinement chain. In fact there is a proof obligation within Event-B requiring certain events from machine $M$ to be enabled in the adjacent refinement machine $M^{\prime}$ [Abr10]. We define this proof obligation referred to as S_NDF in the same way as in [Abr10] but we include explicitly the event $t$ it applies to, the corresponding concrete events, and the new events $N_{i+1}$.

S_NDF: Strong Deadlock Freedom with new events If an abstract event is enabled then either some concrete refinement of it is enabled, or some new event is enabled.

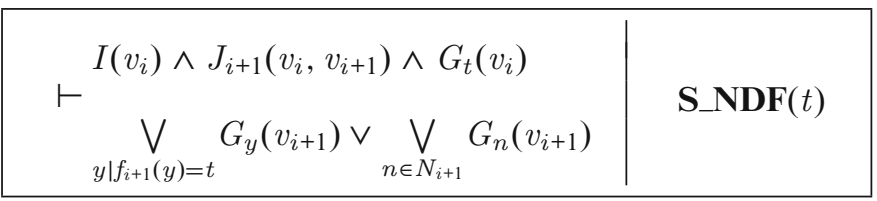

Observe that the refinement from $P M_{0}$ to $P M_{1}$ in Fig. 8 satisfies this proof obligation.

Let us examine this S_NDF proof obligation with respect to all the events in the vending machine development from $V M_{0}$ to $V M_{4}$. Consider again the refinement chain from $V M_{0}$ to $V M_{1}$. In this refinement step S_NDF holds for both the selectItem and the dispenseItem events.

For the refinement step between $V M_{1}$ and $V M_{2}$ S_NDF holds for selectBiscuit, selectChoc, dispenseBiscuit and dispenseChoc since in the refinement step even though the guards are strengthened, the new event pay can occur, hence discharging S_NDF. In the step between $V M_{2}$ and $V M_{3}$ again the new event refill supports the discharging of the S_NDF obligations for all the events in $\alpha\left(V M_{2}\right)$.

In the refinement step from $V M_{3}$ to $V M_{4}$, S_NDF holds vacuously for refund and S_NDF for selectBiscuit, selectChoc, dispenseBiscuit, dispenseChoc, refill simply holds by data refinement. For example in the proof for S_NDF(dispenseBiscuit) the strengthened guard biscuitStock $>0$ is established by the following:

$$
\begin{array}{ll}
J_{4}\left(v_{3}, v_{4}\right): & (\text { biscuit } \in \text { stocked } \Rightarrow \text { biscuitStock }>0) \\
G_{d b}\left(v_{3}\right): & \wedge \text { biscuit } \in \text { stocked } \\
G_{d b}\left(v_{4}\right): & \Rightarrow \text { biscuitStock }>0
\end{array}
$$

In $V M_{4}$ the pay event has a strengthened guard which stops pay happening forever, this is needed in order to turn it into a convergent event but of course this then means that S_NDF does not hold for it since its guard is strengthened and no new events are introduced in $V M_{4}$.

In general, once a machine has an anticipated event that is always enabled, then to make that event convergent its guard will need to be strengthened in a way that fails to meet S_NDF. Therefore, the anticipated approach of deferring convergence proofs is incompatible with the use of S_NDF for each event in all of the refinement steps. Therefore, we need to identify a weaker form of S_NDF. The inspiration for its definition comes from the same principle that we adopt for deadlock freedom, i.e., we do not need deadlock freedom to be a property that is true of each refinement step but it simply needs to hold at the end of a refinement chain. 
Let us therefore also consider S_NDF to be a proof obligation that needs to hold between the refinement step in which an LTL property based on the enabled operator is introduced and the final machine in the refinement chain. It does not need to hold for all the intermediate steps.

We define a new proof obligation referred to as $\mathrm{S}_{-} \mathrm{NDF}_{i, j}(t)$ to relate the enabledness of $t$ in $M_{i}$ with its concrete refinement(s) in $M_{j}$ as follows:

Definition 7.2 If an abstract event is enabled for $M_{i}$ and $i<j$ then either some concrete refinement of it is enabled in $M_{j}$, or some new event is enabled in $M_{j}$.

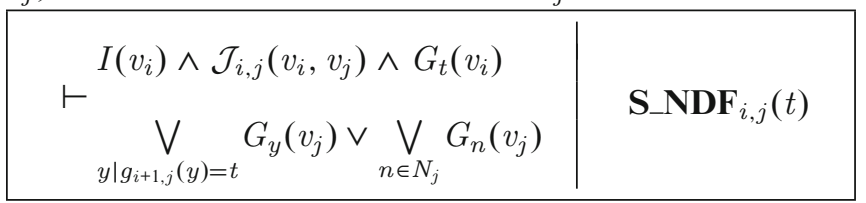

With this extra condition on the preservation of enabledness of events, we are able to extend the main results about the preservation of LTL properties to include the enabled operator (i.e., remove the restriction on its inclusion) as follows:

Lemma 7.3 If $M_{i} \models \phi$ and and $\phi$ does not contain the negation ( $\neg$ ) operator and $M_{i} \preccurlyeq W \quad \cdots \preccurlyeq W \quad M_{n}$ and $0 \leq i<n$ and $\bigwedge_{t \in \phi} \mathrm{S} \_N D F_{i, n}(t)$ and $M_{n}$ is deadlock free and $M_{n}$ does not contain any anticipated events then $M_{n} \vDash$ trans $_{g_{i+1, n}}(\phi)$.

Lemma 7.4 If $M_{i} \models \phi$ and $M_{i} \preccurlyeq{ }_{W} \cdots \preccurlyeq{ }_{W} M_{n}$ and $0 \leq i<n$ and $\bigwedge_{t \in \phi} \mathrm{S} \_N D F_{i, n}(t)$ and the composition of the refinement relations $\mathcal{J}$ is functional and $M_{n}$ is deadlock free and $M_{n}$ does not contain any anticipated events then $M_{n} \models$ trans $_{g_{i+1, n}}(\phi)$.

The proofs of these lemmas is given in Appendix A.

Consider again the property $\phi_{I}$. It is satisfied for $V M_{1}$ and $V M_{2}$, but not for $V M_{3}$, since if biscuit $\notin$ stocked in $V M_{3}$ then selectBiscuit can be followed by an infinite sequence of pay events with no refill event, and so $e$ (dispenseBiscuit) never becomes true. However $\mathrm{S}_{-} \mathrm{NDF}_{1,4}$ holds, $V M_{4}$ has no anticipated events (thus pay cannot occur for ever) and Lemma 7.3 yields that $V M_{4} \models \operatorname{trans}_{g_{2,4}}\left(\phi_{I}\right)$. Expanding out $\operatorname{trans}_{g_{2,4}}\left(\phi_{I}\right)$ simplifies to:

$$
\begin{aligned}
G([\text { selectBiscuit }] \Rightarrow X & (([\text { pay }] \vee[\text { refund }] \vee[\text { refill }]) \\
U( & (\neg e(\text { pay }) \wedge \neg e(\text { refund }) \wedge \neg e(\text { refill }) \wedge e(\text { dispenseBiscuit })) \\
& \vee(e(\text { pay }) \vee e(\text { refund }) \vee e(\text { refill })) \\
& \quad \wedge([\text { selectBiscuit }] \vee[\text { selectChoc }] \vee[\text { dispenseBiscuit }] \vee[\text { dispenseChoc }])))))))
\end{aligned}
$$

This states that, following selectBiscuit, the new events pay, refund and refill may occur until either none of them are enabled any more, and dispenseBiscuit is enabled; or any of the select or dispense might occur while the new events are still enabled. Thus if the customer is prepared to wait for dispenseBiscuit, and does not engage in any of the other events, then dispenseBiscuit will eventually become enabled.

The inclusion of the enabled operator allows for the expression of useful fairness properties. Fairness assumptions are often required when model checking systems for liveness properties, so as to dismiss infinite behaviours (that may violate liveness properties) that are judged to be unfair. Here fair typically means that "if a certain choice is possible sufficiently often, then it is sufficiently often taken" [AFK88]. Strong (resp. weak) event fairness frame fairness properties in the context of event-based formalisms such as Event-B [Lam00, PV01, SLDW08, Mur13]. Given a set of events $\Sigma$, weak event fairness with respect to $\Sigma$ demands that any continually enabled event from $\Sigma$ must occur infinitely often, whereas strong event fairness with respect to $\Sigma$ demands that infinitely often enabled events occur from $\Sigma$ infinitely often.

$$
\begin{aligned}
W E F & =\bigwedge_{z \in \Sigma}(F G e(z) \Rightarrow G F[z]) \\
S E F & =\bigwedge_{z \in \Sigma}(G F e(z) \Rightarrow G F[z])
\end{aligned}
$$

Consider the following new properties, with $\Sigma=\{$ selectChoc, selectBisc, dispenseChoc, dispenseBisc $\}$ :

$\phi_{J}=W E F \Rightarrow G F[$ dispenseChoc $]$ 
Supposing each continually enabled event occurs infinitely often, then dispenseChoc occurs infinitely often.

$$
\phi_{K}=S E F \Rightarrow G F[\text { dispenseChoc }]
$$

Supposing each infinitely often enabled event occurs infinitely often, then dispenseChoc occurs infinitely often.

$V M_{1}$ satisfies both of these properties. One infinite trace that violates $G F[$ dispenseChoc] is the following: $\langle\text { selectBiscuit, dispenseBiscuit }\rangle^{\omega}$. In this trace selectChoc is continuously enabled but never taken and is thus deemed unfair. In fact the only traces that violate the property GF[dispenseChoc] are those that (unfairly) continuously deny either selectChoc or dispenseChoc; GF[dispenseChoc] is satisfied under the assumption of both strong and weak event fairness. From our results we may deduce that dispenseChoc is guaranteed to occur infinitely often under (a translation of) the assumption of weak event fairness and hence is also satisfied in $V M_{4}$.

\section{Related work}

One of the few papers to discuss LTL preservation in Event-B refinement is Groslambert [Gro06]. The LTL properties were defined in terms of predicates on system state where we also include events' occurrences and enableness in our formulation. His paper focused only on the introduction of new convergent events. It did not include a treatment of anticipated events, but this is unsurprising since the paper was published before their inclusion in Event-B. Our results are more general in two ways. Firstly, the results support the treatment of anticipated events. Secondly, we allow more flexibility in the development methodology. A condition of Groslambert's results was that all the machines in the refinement chain needed to be deadlock free. The four main lemmas in our paper: Lemmas 6.3, 6.4, 7.3 and 7.4 do not require each machine in a refinement chain to be deadlock free, only the final machine. It is irrelevant if intermediate $M_{i}$ s deadlock as long as the deadlock is eventually refined away. This is possible by refining away paths to deadlock.

Groslambert deals with new events via stuttering and leaves them as visible events in a trace. This is why the LTL operators he uses do not include the next operator $(X)$. As new events may happen this may violate the $X$ property to be checked. On the other hand Plagge and Leuschel in [PL10] permit the use of the $X$ operator since they treat the inclusion of new events as internal events which are not visible. In this paper we are also able to deal with the next operator.

The notion of verification of temporal properties of both classical and Event-B systems using proof obligations has been considered in many research papers. Abrial and Mussat in an early paper, [AM98], introduced proof obligations to deal with dynamic constraints in classical B. In a more recent paper [HA11] Hoang and Abrial have also proposed new proof obligations for dealing with liveness properties in Event-B. They focus on three classes of properties: existence, progress and persistence, with a view to implementing them in Rodin. Bicarregui et al. in $\left[\mathrm{BAA}^{+} 08\right]$ introduced a temporal concept into events using the guard in the when clause and the additional labels of within and next so that the enabling conditions are captured clearly and separately. However, these concepts are not aligned with the standard Event-B labelling.

In [BDJK00, BDJK01], the authors propose an approach of combining proofs and model-checking verification techniques to reason about properties formulated in Propositional Linear Temporal Logic (dynamic properties). Similar to our approach, the PLTL properties are first specified at the abstract level (and verified by PLTL model-checking), and gradually reformulated during refinement. Several transformation patterns are mentioned with accompanying sufficient conditions that required to be discharged. As a result, the supported properties are restricted to those that are transformable, e.g., dynamic invariants, leads-to, or until properties. In our work, the translation function trans $_{g}$ is total and can be used to transform any property over a number of refinement steps. Moreover, our temporal language is also richer than PLTL, including the events' occurrences and enableness. In [BDJK00], fairness assumptions are required for reasoning about dynamic properties using PLTL model-checking tools. As a result, the transformation of dynamic properties is conditional on the preservation of fairness assumptions which is consider as future work in [BDJK00]. Since fairness assumptions can be encoded using events' enableness and occurrences in our language, they can be transformed as a part of the temporal properties. Furthermore, the transformation of fairness assumptions using our approach can be the basis for future research on preservation of fairness assumptions via refinement.

In [HH13], the Unit-B modelling method is proposed as a combination of UNITY [CM89] and Event$B$ [Abr10]. Unit-B extends the Event-B events with coarse- and fine-schedules, a generalisation of weak- and strong-fairness assumptions, stating how often an event has to occur. Liveness reasoning in Unit-B is borrowed from the UNITY logic and is adapted for the newly introduced scheduling assumptions. Refinement in Unit-B 
amounts to proving that the liveness assumptions are strengthened and the rules are based on reasoning about progress properties.

To compare with our work, the LTL used in Unit-B is defined in term of state variables and does not included references to event occurrences $[t]$ or event enableness $e(t)$. In fact the Unit-B traces are defined as sequence of states whereas we explicitly include executions of events into the definition of traces. Moreover, at the moment, Unit-B only considers superposition refinement, where variables of the abstract machine are retained in the concrete machine. As a result, liveness properties are preserved in LTL "as they are". Our work is more general by allowing data refinement, transforming the liveness properties accordingly via the translation function trans $_{g}$. Another difference is the fact that the preservation of properties in our work can stretch across several refinements, i.e., we can postpone the proof of properties preservation for some refinements, until all the necessary conditions (e.g., deadlock-free, event convergence) can be established. In Unit-B, it is required that all the necessary conditions are discharged at every refinement step, so that all liveness properties are maintained. Note also that Unit-B has a single general refinement rule preserving all liveness properties. With the inclusion of event enableness in our language, we have additional conditions for refinement depending on the properties we want to preserve.

In order to prove liveness properties relying on reasoning about convergence and deadlock-freeness, we made an assumption on event execution for each machine (called minimal progress in [HH13]): if the system is not deadlocked then one of the enabled events will be executed. In Unit-B, the assumption on event execution is finer-grained. Individual scheduling assumptions can be attached to each event. As a result, Unit-B can express stronger fairness assumptions and subsequently can prove more liveness properties relying on these assumptions.

Approaches to LTL preservation through refinement are wider than simply Event-B. For example, Derrick and Smith [DS12] discuss the preservation of LTL properties in the context of Z refinement, extending their results to other logics such as CTL and the $\mu$ calculus. They focus on discussing the restrictions that are needed on temporal-logic properties and retrieve relations to enable the model checking of such properties. Their refinements are restricted to data refinement and do not permit the introduction of new events in refinement steps. Our paper does permit new events to be introduced during refinement steps and the conditions that are required to hold at each refinement step do not provide any restrictions on the properties that can be expressed.

\section{Discussion and future work}

The paper has provided the theoretical foundation to justify the use of LTL properties in Event-B development. It provides foundational results that justify when temporal properties hold at the end of an Event-B refinement chain for developments which contain anticipated, convergent and ordinary events, which goes beyond that presented in [Gro06]. Moreover, the class of LTL operators that is supported by the theory also extends the LTL presented in [Gro06] and are those supported by ProB. Notably, our inclusion of the enabled operator, and its translation in Definition 7.1, (reflecting Lowe's available operator [Low08]) allows for the expression of temporal properties under various notions of fairness [Mur13, WdRF12]. The expression of fairness properties are typically required in proving pertinent liveness properties.

From the results in this paper, a complex (even infinite branching/infinite state) concrete machine can be shown to satisfy such a property by first model checking a small abstract machine using ProB and following a particular refinement strategy, discharging the proofs in Rodin or Atelier B.

The paper has removed one of the awkward restrictions of our previous work, which imposed restrictions on the temporal properties in terms of being $\beta$-dependent, which determined when a temporal property of interest should be introduced into the development chain. 


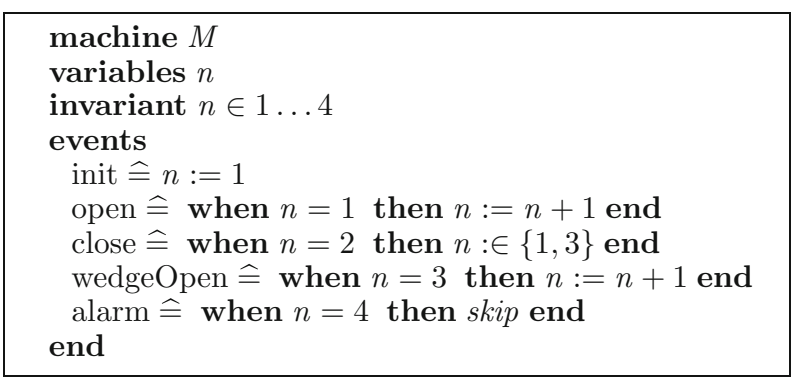

Fig. 10. $M$

In this paper we restricted two of the lemmas, Lemmas 6.4 and 7.4, to refer to developments whose linking invariants were required to be functional in order to deal with negation in LTL properties. Event-B developments generally contain functional invariants, since abstract machines usually contain less information than their refinements, and thus functional mappings in this case make sense. There are of course examples (e.g., the alternating bit protocol of [Abr10]) where this is not the case, but these are rare.

The novelty of the results, which is a clear extension from [STWW14b], is the requirement to establish strong deadlock freedom S_NDF, in certain circumstances, in order to guarantee the preservation of LTL properties that include the enabled operator. The conditions of the lemmas required to hold and the proof obligations required lend themselves to the practical application of Event-B. We are looking into how to provide the new S_NDF rule over a number of refinement steps in terms of chaining single step S_NDF proof obligations for the corresponding events, so that this proof obligation can be tool supported.

The proofs in this paper were defined on infinite traces. The proofs are not defined in terms of a CSP semantics model. Our work in [STW14] provided a CSP traces divergences and infinite traces semantics for Event-B. Our ongoing work is combining these results with those in this paper in order to provide a cohesive process algebra underpinning for Event-B and the preservation of LTL properties. It should not be surprising that the underpinning will be in terms of the refusal traces semantic model of CSP; Definitions 6.2 and 7.1 were based on [WdRF12, Low08], which addressed the preservation of LTL properties through refusal traces refinement. (In [Low08] temporal operators were defined in terms of the set of finite and infinite refusal traces they allow.)

We have focused in this paper on a refinement strategy that achieved convergence of all its new events by the end. We could also consider the impact on temporal property preservation in refinement chains where anticipated events remain present in the final machine. We have also yet to deal with extending the work to merging events in a refinement chain. Consider the example in Fig. 10 the events open and wedgeOpen meet the conditions to allow them to be merged. In particular they have the same body $n:=n+1$. The LTL property $G$ (open $\Rightarrow F($ close)) is satisfied by $M$. However, if open and wedgeOpen are merged then this property no longer holds. Hence, the results of the paper do not apply. A different approach would be required.

\section{Acknowledgements}

Thanks to Thierry Lecomte for discussions about Event-B and its practical use. We are also grateful to the reviewers for their thorough and detailed comments on this paper.

Open Access This article is distributed under the terms of the Creative Commons Attribution 4.0 International License (http://creativecommons.org/licenses/by/4.0/), which permits unrestricted use, distribution, and reproduction in any medium, provided you give appropriate credit to the original author(s) and the source, provide a link to the Creative Commons license, and indicate if changes were made.

\section{Appendix A: Proofs of Lemmas}

In this appendix we provide the proofs for the main lemmas: Lemmas 6.1, 6.3, 6.4, 7.3 and 7.4. We also provide additional supporting definitions and lemmas.

Proof of Lemma 6.1 This proof is restated from [STWW14a] Since $M_{n}$ does not deadlock consider an infinite trace $u$ of $M_{n}$. Let $u=u \uparrow\left(C_{1} \cup \cdots \cup C_{n} \cup O_{0}\right)$ since $M_{n}$ does not have any anticipated events. We aim to prove that $u \uparrow O_{0}$ is infinite. If $u \uparrow C_{1} \cup \cdots \cup C_{n}$ is finite then $u \uparrow O_{0}$ is infinite. If $u \uparrow C_{1} \cup \cdots \cup C_{n}$ is infinite then 
$u \uparrow O_{0}$ is infinite by Theorem 4.3. Hence, since $u \uparrow O_{0}$ is infinite then the occurrence of one of the events in $M_{0}$ will be always eventually be possible.

The following lemmas: Lemmas A.1 and A.2, provide results that allow us to demonstrate the relationship between an infinite trace $u_{a}$ preserving a temporal property and a corresponding trace $u_{c}$ preserving a transformed temporal property. The two lemmas are subtly different and both are required due to the subtleties in the proof when using the proposition and negation operators. The lemmas are required in order to prove Lemmas 6.3 and 6.4 presented in Sect. 6 and proved below.

Lemma A.1 If $\phi$ does not contain the enabled $e$ and the negation $\neg$ operators and $\alpha(\phi) \subseteq \operatorname{ran}(g)$ and $u_{a} R u_{c}$ then $u_{a} \models \phi \Rightarrow u_{c} \models \operatorname{trans}_{g}(\phi)$

Proof By structural induction over the structure of $\phi$.

Base Cases:

Consider where $\phi$ is true. Trivially holds.

Consider where $\phi$ is $[t]$ : Let $u_{a} R u_{c}$ and $u_{a}=\left\langle s_{0}, t_{0}, s_{1}, t_{1}, \ldots\right\rangle$

If $u \models[t]$ then $t_{0}=t$ by definition of $[t]$

Since $u_{c} \uparrow \operatorname{dom}(g)$ is infinitethenthere is a first $t_{k}^{\prime}$ such that $t_{k}^{\prime} \in \operatorname{dom}(g)$.

So $g\left(t_{k}^{\prime}\right)=t_{0}$ since $u_{a} R u_{c} \wedge \forall j<k . t_{j}^{\prime} \in N$

So $u_{c} \models \bigvee_{n \in N}[n] U \bigvee_{y \mid g(y)=t}[y]$

So $u_{c} \models \operatorname{trans}_{g}([t])$ by trans definition

Consider where $\phi$ is $p$ : If $u_{a} \models \phi$ then $p$ is true in $s_{0}$ by definition $\mathcal{J}\left(s_{0}, s_{0}^{\prime}\right)$ holds from the definition of $u_{a} R u_{c}$ $\Rightarrow u_{c} \vDash \exists s_{0} \cdot \mathcal{J}\left(s_{0}, s_{0}^{\prime}\right) \wedge p \in s_{0}$.

Inductive Cases:

Case $\phi_{1} \vee \phi_{2}$ :

$$
\begin{aligned}
u_{a} & \models \phi_{1} \vee \phi_{2} \\
& \Rightarrow u_{a} \models \phi_{1} \vee u_{a} \models \phi_{2} \\
& \Rightarrow u_{c} \models \operatorname{trans}_{g}\left(\phi_{1}\right) \vee u_{c} \models \operatorname{trans}_{g}\left(\phi_{2}\right) \\
& \Rightarrow u_{c} \models \operatorname{trans}_{g}\left(\phi_{1} \vee \phi_{2}\right)
\end{aligned}
$$

Same for $\phi_{1} \wedge \phi_{2}$.

Case $\phi_{1} U \phi_{2}$ :

If $u_{a} \models \phi_{1} U \phi_{2} \Leftrightarrow \exists k \geq 0 . \forall i<k . u_{a}^{i} \models \phi_{1} \wedge u_{a}^{k} \models \phi_{2}$ by definition of $U$.

$k$ is the $k^{\text {th }}$ state of $u_{a}$ and $\phi_{2}$ holds for the path starting from that state, the point where $\phi_{2}$ is reached.

Let $l$ be the least value such that $u_{a}^{k} R u_{c}^{l}$ holds. Then $\forall j<l . u_{c}^{j} \exists i<k . u_{a}^{i} R u_{c}^{j}$. Then $u_{a}^{i} \models \phi_{1}$, so by ind hyp $u_{c}^{j} \models \operatorname{trans}_{g}\left(\phi_{1}\right)$. Also since $u_{a}^{k} \models \phi_{2}$ by ind hyp $u_{c}^{l} \models \operatorname{trans}_{g}\left(\phi_{2}\right)$. Therefore, $u_{c} \models \operatorname{trans}_{g}\left(\phi_{1} U \phi_{2}\right)$.

Case $X \phi$ : If $u_{a} \models X \phi$ and $u_{a}=\left\langle s_{0}, t_{0}, s_{1}, t_{1}, \ldots\right\rangle$ then $u_{a}^{1}=\left\langle s_{1}, t_{1}, \ldots\right\rangle \models \phi$ by definition of $X$.

Let $u_{a} R u_{c}$. Since $u_{c}\left\lceil\operatorname{dom}(g)\right.$ is infinite then there is a first $t_{k}^{\prime}$ such that $t_{k}^{\prime} \in \operatorname{dom}(g)$.

Let $u_{c}=\left\langle s_{0}^{\prime}, t_{0}^{\prime}, \ldots, s_{k-1}^{\prime}, t_{k-1}^{\prime}\right\rangle \frown\left\langle s_{k}^{\prime}, t_{k}^{\prime}, s_{k+1}^{\prime}, t_{k+1}^{\prime}, \ldots\right\rangle$.

So $u_{a}^{1} R u_{c}^{k+1}$ and by ind hyp $u_{a}^{1} \models \phi \Rightarrow u_{c}^{k+1} \models \operatorname{trans}_{g}(\phi)$.

Since $\forall i<k \quad u_{c}^{i} \models \bigvee_{n \in N}[n] \wedge u_{c}^{k} \models \bigvee_{d \in \operatorname{dom}(g)}[d] \wedge X\left(\right.$ trans $\left._{g}(\phi)\right)$ since $t_{k}^{\prime} \in \operatorname{dom}(g)$ by definition of $X$ for $u_{c}^{k}$. Therefore $\exists k \geq 0 . \forall i<k . u_{c}^{i} \models \bigvee_{n \in N}[n] \wedge\left(u_{c}^{k} \models \bigvee_{d \in \operatorname{dom}(g)}[d] \wedge X\left(\right.\right.$ trans $\left.\left._{g}(\phi)\right)\right)$. Hence by definition of trans $g$ $u_{c} \models \operatorname{trans}_{g}(\phi)$.

Lemma A.2 If $\phi$ does not contain the enabled e operator and $\alpha(\phi) \subseteq \operatorname{ran}(g)$ and $u_{a} R u_{c}$ and $\mathcal{J}$ is functional then $u_{a} \vDash \phi \Leftrightarrow u_{c} \models \operatorname{trans}_{g}(\phi)$

Proof By structural induction over the structure of $\phi$.

Base Cases:

Consider where $\phi$ is true. Trivially holds. 
Consider where $\phi$ is $[t]$ :

Consider " $\Rightarrow$ " as in Lemma A.1.

Consider " $\Leftarrow$ ": Let $u_{a} R u_{c}$ and $u_{a}=\left\langle s_{0}, t_{0}, s_{1}, t_{1}, \ldots\right\rangle$

$$
\text { If } \begin{aligned}
u_{c} & \models \bigvee_{n \in N}[n] U \bigvee_{y \mid g(y)=t}[y] \\
& \Rightarrow \exists k \geq 0 . \forall i<k . u_{c}^{i} \models \bigvee_{n \in N}[n] \wedge u_{c}^{k} \models \bigvee_{y \mid g(y)=t}[y] \text { (definition of } U \text { ) } \\
& \Rightarrow i<k \Rightarrow \bigvee_{n \in N}\left(t_{i}^{\prime}=n\right) \wedge t_{k}^{\prime} \in\{y \mid g(y)=t\} \\
& \Rightarrow i<k . t_{i}^{\prime} \notin \operatorname{dom}(g) \wedge t_{k}^{\prime} \in \operatorname{dom}(g) \wedge g\left(t_{k}^{\prime}\right)=t=t_{0} \\
& \Rightarrow u_{a} \models[t]
\end{aligned}
$$

Consider where $\phi$ is $p$ :

Consider" $\Rightarrow$ " as in Lemma A.1.

Consider " $\Leftarrow$ ": If $u_{c} \models \exists ! s_{o} . \mathcal{J}\left(s_{0}, s^{\prime}\right) \wedge p \in s_{0}$, since $\mathcal{J}$ is functional. Now consider $u_{a} R u_{c}$ then $u_{a}$ has an initial state $s_{0}$ which is related through $\mathcal{J}$ to the concrete state and therefore $p$ is true in $s_{0}$, thus $u_{a} \models p$.

Inductive Cases:

Case $\neg \phi$ :

$$
\begin{aligned}
u_{a} & \models \neg \phi \\
& \Leftrightarrow \neg u_{a} \models \phi \\
& \Leftrightarrow \neg\left(u_{c} \models \operatorname{trans}_{g}(\phi)\right) \text { by ind hyp and requires } \Leftrightarrow \\
& \Leftrightarrow u_{c} \models \neg \operatorname{trans}_{g}(\phi) \\
& \Leftrightarrow u_{c} \models \operatorname{trans}_{g}(\neg \phi)
\end{aligned}
$$

Case $\phi_{1} \vee \phi_{2}$ : Similar to Lemma A.1 with $\Rightarrow$ replaced by $\Leftrightarrow$ in the steps.

Same for $\phi_{1} \wedge \phi_{2}$.

Case $\phi_{1} U \phi_{2}$ :

Consider " $\Rightarrow$ " as in Lemma A.1.

Consider " $\models$ ": If $u_{c} \models \operatorname{trans}_{g}\left(\phi_{1} U \phi_{2}\right)$ then $u_{c} \models \operatorname{trans}_{g}\left(\phi_{1}\right) U \operatorname{trans}_{g}\left(\phi_{2}\right)$ by definition of $\operatorname{trans}_{g}(U)$.

Hence $\exists k \geq 0 . \forall i<k . u_{c}^{i} \models \operatorname{trans}_{g}\left(\phi_{1}\right) \wedge u_{c}^{k} \models \operatorname{trans}_{g}\left(\phi_{2}\right)$ by definition of $U$.

Let $l$ be the least value such that $u_{a}^{l} R u_{c}^{k}$ holds. Then $\forall j<l . u_{a}^{j} \vDash \phi_{1} \wedge u_{a}^{l} \vDash \phi_{2}$ by ind hyp. Therefore, $u_{a} \models \phi_{1} U \phi_{2}$.

Case $X \phi$ :

Consider " $\Rightarrow$ " as in Lemma A.1.

Consider " $\Leftarrow$ ": If $u_{c} \models \operatorname{trans}_{g}(X \phi)$ then $u_{c} \models \bigvee_{n \in N}[n] U\left(\bigvee_{d \in \operatorname{dom}(g)}[d] \wedge X \operatorname{trans}_{g}(\phi)\right)$ by definition of $\operatorname{trans}_{g}(X \phi)$ $\Leftrightarrow \exists k \geq 0 . \forall i<k . u_{c}^{i} \models \bigvee_{n \in N}[n] \wedge u_{c}^{k} \models \bigvee_{d \in \operatorname{dom}(g)}[d] \wedge X$ trans $_{g}(\phi)$ by definition of $U$.

Let $u_{c}=\left\langle s_{0}^{\prime}, t_{0}^{\prime}, s_{1}^{\prime}, t_{1}^{\prime}, \ldots, s_{k}^{\prime}, t_{k}^{\prime}, \ldots\right\rangle$. So $t_{i} \in N \forall i<k, t_{k} \in \operatorname{dom}(g)$ and $u_{c}^{k+1}=\operatorname{trans}_{g}(\phi)$ by the definition of $X)$.

Also given $u_{a} R u_{c}$ then $u_{a}^{1} R u_{c}^{k+1}$ then $u_{a}^{1} R u_{c}^{k+1}$ by ind hyp $u_{a}^{1} \models \phi$ therefore $u_{a} \models X \phi$.

The next lemma allows us to determine that there is a relationship between infinite concrete paths, which are infinite due to convergent and ordinary (and not anticipated) events, and an abstract path such that the refinement relation $R$ introduced in Definition 4.1 holds.

Lemma A.3 If $M \preccurlyeq W \cdots \preccurlyeq W M^{\prime}$ and $u^{\prime}$ is an infinite path of $M^{\prime}$ with no anticipated events, then $\exists$ u.u is a path of $M$ and $u R u^{\prime}$.

Proof Let $u^{\prime}=\left\langle s_{0}^{\prime}, t_{0}^{\prime}, s_{1}^{\prime}, t_{1}^{\prime}, \ldots\right\rangle$

$$
\begin{aligned}
& \left.r\left(s_{0}, u^{\prime}\right)=\left\langle s_{0}, g\left(t_{0}^{\prime}\right)\right\rangle\right\urcorner r\left(s_{1},\left\langle s_{1}^{\prime}, t_{1}^{\prime}, \ldots\right\rangle\right) \text { if } t_{0}^{\prime} \in \operatorname{dom}(g) \text { for } s_{1} \text { where } \mathcal{J}\left(s_{1}, s_{1}^{\prime}\right) \\
& r\left(s_{0}, u^{\prime}\right)=r\left(s_{0},\left\langle s_{1}^{\prime}, t_{1}^{\prime}, \ldots\right\rangle\right) \text { if } t_{0}^{\prime} \notin \operatorname{dom}(g) \text { where } \mathcal{J}\left(s_{0}, s_{1}^{\prime}\right)
\end{aligned}
$$


Let $u=r\left(s_{0}, u^{\prime}\right)$. then by construction $u R u^{\prime}$.

Proof of Lemma 6.3 Let $u^{\prime}$ to be an infinite execution of $M_{n}$ since $M_{n}$ is deadlock free then $M_{n}$ sat $C A\left(g_{i+1, n}^{-1}\left(C_{i-1}\right) \cup\right.$ $\left.\cdots \cup C_{n}, g_{i+1, n}^{-1}\left(O_{i}\right)\right)$ thus $u^{\prime}\left\lceil\operatorname{dom}\left(g_{i+1, n}\right)\right.$ is infinite since $M_{n}$ has no anticipated events.

From Lemma A.3 $u$ is a path of $M_{i}$ such that $u R u^{\prime}$.

Since $M_{i} \models \phi$ then $u \models \phi$. Thus, from Lemma A.1 $u^{\prime} \models \operatorname{trans}_{g_{i+1, n}}(\phi)$ thus $M_{n} \models \operatorname{trans}_{g_{i+1, n}}(\phi)$ as required.

Note that $u^{\prime}\left\lceil\operatorname{dom}\left(g_{i, n}\right)\right.$ being infinite is key within the proof in order to be able to establish the conditions for $R$ to hold. Recall the example of $u_{1}=\langle a, a, a, a, \ldots\rangle$ and $u_{2}=\langle b, c, b, c, b, c, c, c, c, c, c, \ldots\rangle$ where $b$ maps to $a$, and $c$ is a new event. $u_{1} \models G F(a)$ but $u_{2} \not \models G F(b)$, i.e. the transformed property is not satisfied by $u_{2}$ since $u_{2}$ only has finitely many events in $u_{1}$ that it matched.

Proof of Lemma 6.4 The proof of this lemma is almost identical to Lemma 6.3, noting that the existence of a path does not rely on the fact that $\mathcal{J}$ is functional. But since $\mathcal{J}$ is functional the path will be unique and we are able to apply Lemma A.2 in the penultimate step so that $u^{\prime} \models \operatorname{trans}_{g_{i+1, n}}(\phi)$ thus $M_{n} \models \operatorname{trans}_{g_{i+1, n}}(\phi)$ as required.

The remainder of the appendix provides the proofs that support the most general results in the paper, i.e., Lemmas 7.3 and 7.4. We begin as above by providing a supporting lemma, Lemma A.4 to demonstrate the relationship between an infinite trace $u_{a}$ preserving a temporal property which refers to the enabled operator and a corresponding trace $u_{c}$ preserving a transformed temporal property. In this lemma we have been more explicit with our labelling with regard to which refinement steps are being used, i.e., between $M_{i}$ and $M_{j}$, thus it is clearer between which refinement steps the strong deadlock freedom proof obligation needs to hold. We have also labelled the compositional function mapping $g$ more explicitly for consistency within the proof.

Lemma A.4 If $u_{a}$ is a path of $M_{i}$ and $u_{c}$ is a path of $M_{j}$ such that $u_{a} R u_{c}$ and $u_{a} \models e(t)$ and $u_{c}\left\lceil\operatorname{dom}\left(g_{i+1, j}\right)\right.$ is infinite and $\mathrm{S} \_\mathrm{NDF}_{i, j}(t)$ holds $\Rightarrow u_{c}=$ trans $_{g_{i+1, j}}(e(t))$.

Proof Consider $u_{a} R u_{c}$ such that $u_{c}=\left\langle s_{0}^{\prime}, t_{0}^{\prime}, s_{1}^{\prime}, t_{1}^{\prime}, \ldots\right\rangle$ and $u_{a}=\left\langle s_{0}, t_{0}, \ldots\right\rangle$.

Then let $t_{0}^{\prime}, t_{1}^{\prime}, \ldots, t_{k-1} \in N$ and $t_{k}^{\prime} \notin N$, i.e., $t_{k}^{\prime} \in \operatorname{dom}\left(g_{i+1, j}\right)$ and $N=\alpha\left(M_{j}\right)-\operatorname{dom}\left(g_{i+1, j}\right)$

Then $\forall i<k . u_{c}^{i}=\bigvee_{n \in N}[n]$

Thus from Definition 7.1 we are required to prove that

$$
u_{c}^{k} \models\left(\left(\bigwedge_{n \in N} \neg e(n) \wedge \bigvee_{y \mid g_{i+1, j}(y)=t} e(y)\right) \vee\left(\bigvee_{n \in N} e(n) \wedge \bigvee_{d \in \operatorname{dom}\left(g_{i+1, j}\right)}[d]\right)\right)
$$

Note that $u_{a} \models e(t) \Rightarrow G_{t}\left(s_{0}\right)$.

We have $\mathcal{J}\left(s_{0}, s_{k}^{\prime}\right)$ since $t_{i} \in N, i<k$ and $u_{a} R u_{c}$.

Since S_NDF $i, j(t)$ we have by Definition 7.2 that $\mathcal{J}\left(s_{0}, s_{k}^{\prime}\right) \wedge G_{t}\left(s_{0}\right) \Rightarrow \bigvee_{y \mid g_{i+1, j}(y)=t} e(y)\left(s_{k}^{\prime}\right) \vee \bigvee_{n \in N} e(n)\left(s_{k}^{\prime}\right)$ It follows that $\bigvee_{y \mid g_{i+1, j}(y)=t} e(y)\left(s_{k}^{\prime}\right) \vee \bigvee_{n \in N} e(n)\left(s_{k}^{\prime}\right)$

$$
\left.\Rightarrow\left(\bigvee_{y \mid g_{i+1, j}(y)=t} e(y)\left(s_{k}^{\prime}\right) \wedge \neg \bigvee_{n \in N} e(n)\left(s_{k}^{\prime}\right)\right) \vee \bigvee_{n \in N} e(n)\left(s_{k}^{\prime}\right) \quad(\text { since } A \vee B=(A \wedge \neg B) \vee B)\right)
$$

$$
\text { Also } \bigvee_{d \in \operatorname{dom}\left(g_{i+1, j}\right)}[d]\left(s_{k}^{\prime}\right)
$$

$$
\text { by construction of } s_{k}^{\prime} \text {, i.e., } t_{k}^{\prime} \in \operatorname{dom}\left(g_{i+1, j}\right)
$$

Thus for $s_{k}^{\prime}$ we have (18) and (19) $\Rightarrow$

$$
\begin{aligned}
& \left(\bigvee_{y \mid g_{i+1, j}(y)=t} e(y)\left(s_{k}^{\prime}\right) \wedge \neg \bigvee_{n \in N} e(n)\left(s_{k}^{\prime}\right)\right) \\
& \left.\quad \times\left(\bigvee_{n \in N} e(n)\left(s_{k}^{\prime}\right) \wedge \bigvee_{d \in \operatorname{dom}\left(g_{i+1, j}\right)}[d]\left(s_{k}^{\prime}\right)\right) \quad \text { since }(A \wedge \neg B) \vee B\right) \wedge C \Rightarrow(A \wedge \neg B) \vee(B \wedge C)
\end{aligned}
$$


i.e.

$$
u_{c}^{k} \models\left(\bigvee_{y \mid g_{i+1, j}(y)=t} e(y) \wedge \neg \bigvee_{n \in N} e(n)\right) \vee\left(\bigvee_{n \in N} e(n) \wedge \bigvee_{d \in \operatorname{dom}\left(g_{i+1, j}\right)}[d]\right)
$$

Thus $u_{c} \models \bigvee_{n \in N}[n] U(20)$.

Proof of Lemma 7.3 Let $u^{\prime}$ to be an infinite execution of $M_{n}$ since $M_{n}$ is deadlock free then $M_{n}$ sat $C A\left(g_{i+1, n}^{-1}\left(C_{i}\right) \cup\right.$ $\left.\cdots \cup C_{n}, g_{i+1, n}^{-1}\left(O_{i}\right)\right)$ thus $u^{\prime} \uparrow d o m\left(g_{i+1, n}\right)$ is infinite since $M_{n}$ has no anticipated events.

From Lemma A.3 $u$ is a path of $M_{i}$ such that $u R u^{\prime}$.

Since $M_{i} \models \phi$ then $u \models \phi$. We also have that $\mathrm{S} \_N D F_{i, n}(t)$ holds, thus from Lemma A.4 (with $i=i$ and $j=n$ ) and Lemma A.1 we have that $u^{\prime} \models \operatorname{trans}_{g_{i+1, n}}(\phi)$ thus $M_{n} \models \operatorname{trans}_{g_{i+1, n}}(\phi)$ as required.

Proof of Lemma 7.4 This proof is identical to the proof of Lemma 7.3 but with the penultimate step applying Lemma A. 2 since $\mathcal{J}$ is functional.

\section{References}

$\left[\mathrm{ABH}^{+} 10\right] \quad$ Abrial J, Butler MJ, Hallerstede S, Hoang TS, Mehta F, Voisin L (2010) Rodin: an open toolset for modelling and reasoning in Event-B. STTT 12(6):447-466

[Abr10] Abrial J-R (2010) Modeling in Event-B: system and software engineering. Cambridge University Press, Cambridge

[AFK88] Apt KR, Francez N, Katz S (1988) Appraising fairness in languages for distributed programming. Distrib Comput 2(4):226-241

[AM98] Abrial J, Mussat L (1998) Introducing dynamic constraints in B. In: Bert D (ed) B'98: Recent Advances in the Development and Use of the B Method, vol. 1393. Springer, Heidelberg, pp 83-128

[BAA ${ }^{+}$08] Bicarregui J, Arenas A, Aziz B, Massonet P, Ponsard C (2008) Towards modelling obligations in Event-B. In: Börger E, Butler M, Bowen JP, Boca P (eds) Abstract state machines, B and Z, vol. 5238. Springer, Heidelberg, pp 181-194

[BDJK00] Bellegarde F, Darlot C, Julliand J, Kouchnarenko O (2000) Reformulate dynamic properties during B refinement and forget variants and loop invariants. In: Bowen JP, Dunne S, Galloway A, King S (eds) ZB 2000: Formal specification and development in Z and B. First International Conference of B and Z Users, York, UK, August 29-September 2, 2000, Proceedings, volume 1878 of Lecture notes in computer science. Springer, pp 230-249

[BDJK01] Bellegarde F, Darlot C, Julliand J, Kouchnarenko O (2001) Reformulation: a way to combine dynamic properties and B refinement. In: Oliveira JN, Zave P (eds) FME 2001: Formal methods for increasing software productivity, International Symposium of Formal Methods Europe, Berlin, Germany, March 12-16, 2001, Proceedings, volume 2021 of Lecture notes in computer science. Springer, pp 2-19

[But92] Butler MJ (1992) A CSP approach to action systems. DPhil thesis, Oxford University

[Cle14] ClearSy (2014) Atelier B version 4.2. http://www.atelierb.eu/en/download-atelier-b/. Accessed 20 Feb 2016

[CM89] Chandy M, Misra J (1989) Parallel program design: a foundation. Addison-Wesley, Reading, Mass

[DJK03] Darlot C, Julliand J, Kouchnarenko O (2003) Refinement preserves PLTL properties. In: ZB 2003: Formal specification and development in $Z$ and B, Third International Conference of B and Z Users, Turku, Finland, June 4-6, 2003, Proceedings, pp 408-420

[DS12] Derrick J, Smith G (2012) Temporal-logic property preservation under Z refinement. Form Asp Comput 24(3):393-416

[Gro06] Groslambert J (2006) Verification of LTL on B event systems. In: B 2007: Formal specification and development in B, volume 4355 of LNCS. Springer, pp 109-124

[HA11] Hoang TS, Abrial J (2011) Reasoning about liveness properties in Event-B. In: ICFEM, volume 6991 of LNCS. Springer, pp 456-471

[HH13] Hudon S, Hoang TS (2013) Systems design guided by progress concerns. In: Integrated formal methods—10th International Conference on integrated formal methods (IFM2013), volume 7940 of Lecture notes in computer science. Springer-Verlag, pp $16-30$

[HLP13] Hallerstede S, Leuschel M, Plagge D (2013) Validation of formal models by refinement animation. Sci Comput Program 78(3):272-292

[Lam00] Lamport L (2000) Fairness and hyperfairness. Distrib Comput 13(4):239-245

[LB08] Leuschel M, Butler MJ (2008) ProB: an automated analysis toolset for the B method. STTT 10(2):185-203

[LFFP09] Leuschel M, Falampin J, Fritz F, Plagge D (2009) Automated property verification for large scale B models. In: FM, volume 5850 of LNCS. Springer, pp 708-723

[Low08] Lowe G (2008) Specification of communicating processes: temporal logic versus refusals-based refinement. Form Asp Comput 20(3):277-294

[Mor90] Morgan CC (1990) Of wp and CSP. In: Feijen WHJ et al (eds) Beauty is our business: a birthday salute to E. W. Dijkstra. Springer, New York, pp 319-326

[Mur13] Murray TC (2013) On the limits of refinement-testing for model-checking CSP. Form Asp Comput 25(2):219-256

[PL10] Plagge D, Leuschel M (2010) Seven at one stroke: LTL model checking for high-level specifications in B, Z, CSP, and more. STTT 12(1):9-21

[PV01] Puhakka A, Valmari A (2001) Liveness and fairness in process-algebraic verification. In: CONCUR 2001—concurrency theory, 12th International Conference, Aalborg, Denmark, August 20-25, 2001, Proceedings, pp 202-217 
[SLDW08] Sun J, Liu Y, Dong JS, Wang HH (2008) Specifying and verifying event-based fairness enhanced systems. In: Formal methods and software engineering, 10th International Conference on formal engineering methods, ICFEM 2008, Kitakyushu-City, Japan, October 27-31, 2008. Proceedings, pp 5-24

[STW14] Schneider S, Treharne H, Wehrheim H (2014) The behavioural semantics of Event-B refinement. Form Asp Comput 26(2):251280

[STWW14a] Schneider S, Treharne H, Wehrheim H, Williams D (2014) Managing LTL properties in Event-B refinement. arXiv:1406.6622, IFM2014, June

[STWW14b] Schneider S, Treharne H, Wehrheim H, Williams DM (2014) Managing LTL properties in Event-B refinement. In: Integrated formal methods - 11th International Conference, IFM 2014, Bertinoro, Italy, September 9-11, 2014, Proceedings, volume 8739 of Lecture notes in computer science. Springer, pp 221-237

[WdRF12] Williams DM, de Ruiter J, Fokkink W (2012) Model checking under fairness in ProB and its application to fair exchange protocols. In ICTAC, volume 7521 of LNCS. Springer, pp 168-182

$\left[\mathrm{Z}^{+}\right.$14] Zurich ETH et al (2014) Rodin version 3.1.0. http://www.event-b.org/. Accessed 20 Feb 2016

Received 25 February 2015

Accepted in revised form 29 March 2016 by Michael Butler

Published online 27 April 2016 